\title{
Understanding XCP: Equilibrium and Fairness
}

\author{
Lachlan L. H. Andrew, Senior Member, IEEE, Steven H. Low, Fellow, IEEE, and Bartek P. Wydrowski, Member, IEEE
}

\begin{abstract}
We prove that the XCP equilibrium solves a constrained max-min fairness problem by identifying it with the unique solution of a hierarchy of optimization problems, namely those solved by max-min fair allocation, but solved by XCP under an additional constraint. This constraint is due to the "bandwidth shuffling" necessary to obtain fairness. We describe an algorithm to compute this equilibrium and derive a lower and upper bound on link utilization. While XCP reduces to max-min allocation at a single link, its behavior in a network can be very different. We illustrate that the additional constraint can cause flows to receive an arbitrarily small fraction of their max-min fair allocations. We confirm these results using ns2 simulations.
\end{abstract}

Index Terms-Congestion control, max-min, optimization.

\section{INTRODUCTION}

$\mathbf{T}$ CP congestion control [1] has prevented severe congestion while the Internet has undergone explosive growth. However, as the network continues to scale in size and capacity, the algorithm is struggling with sensitivity to routine packet loss, slow convergence and low throughput [2], [3]. This has motivated several recent enhancements [4]-[10]. (See [6] for further references.) Of these, XCP [9], [10] has received much attention [11]-[15] and is especially suitable for private networks where its need for explicit communication between the traffic sources and the network is less of a deployment barrier than in the current Internet. Unlike proposals that set the flow rates according to the sum of congestion measures at the links of their paths, XCP sets them according to the minimum "available capacity" in their paths. This has the same flavor as MaxNet [16], [17], which sets flow rates according to the maximum of congestion measures in their paths.

The stability of XCP has been studied when all round-trip times (RTTs) are equal [9], [11] and when they differ [13]. Specific networks can also be shown to be stable [18]. However, that work focuses on very simple topologies. This paper takes the complementary approach [19], [20] of reverse engineering XCP to understand its multi-link equilibrium properties.

Manuscript received February 27, 2008; revised October 24, 2008; approved by IEEE/ACM TRANSACTIONS ON NETWORKING Editor S. Shakkottai. First published August 18, 2009; current version published December 16, 2009. This work was supported by NSF Grant 0303620 . Partial results have appeared in the Proceedings of IEEE INFOCOM, 2005.

L. L. H. Andrew is with Swinburne University of Technology, Hawthorn, Vic. 3122, Australia (e-mail: 1.andrew@ieee.org).

S. H. Low is with the California Institute of Technology, Pasadena, CA 91125 USA (e-mail: slow@ caltech.edu).

B. P. Wydrowski is with Google Inc., New York, NY 10018 USA (e-mail: bwydrowski@google.com).

Color versions of one or more of the figures in this paper are available online at http://ieeexplore.ieee.org.

Digital Object Identifier 10.1109/TNET.2009.2013242
Although packet networks seldom achieve equilibrium, equilibrium analysis indicates the best-case fairness and capacity properties of a protocol. Moreover, dynamic analysis typically starts with a linearization around the equilibrium, which requires a clear characterization such as is presented here. We do not consider misbehaving nodes, as studied in [14], or errors in capacity estimates, as studied in [11].

A deterministic fluid model of a general XCP network with multiple links and multiple flows is presented in Section II. Section III analyzes the equilibrium rates of XCP and shows that all queues are empty in equilibrium We prove the existence and uniqueness of XCP equilibrium rates by identifying them with the unique solution to a hierarchy of optimization problems. This is the same set of problems solved by the standard max-min fair allocation [21], but XCP solves them under an additional constraint. While XCP reduces to max-min allocation at a single link, its behavior in a network can be very different. We describe an algorithm to compute this equilibrium and derive upper and lower bounds on link utilization.

In Section IV, we use these bounds to investigate the impact of the choice of protocol parameters on link utilization under the additional constraint. We show that flows can receive an arbitrarily small fraction of their max-min fair allocations. Specifically, with a max-min fair allocation, as long as a link is a bottleneck for some (not necessarily all) flows that pass through it, it will be fully utilized. Under XCP, this is no longer true: When the majority of flows using a link are bottlenecked at other links, the remaining flows at that link may not fully use the residual bandwidth. With the parameters specified in [9], however, link utilization is at least $80 \%$ at any link. XCP has a "shuffling parameter" $\gamma>0$ to prevent the network from settling into an unfair state [9]. We show that, given any network topology, we can choose $\gamma$ sufficiently small so that the resulting allocation is close to max-min fairness. For any fixed $\gamma>0$, however, there are topologies in which some flow rates can be far away from their max-min allocations.

These properties and the accuracy of our algorithm are verified by ns 2 simulations in Section V. We conclude in Section VI with limitations of this work.

\section{MODEL}

Consider a network with $L$ links shared by $N$ flows. Sources are indexed by $i=1, \ldots, N$, links by $l=1, \ldots, L$ and packets by $k$. Let $R$ be the $L \times N$ routing matrix: $R_{l i}=1$ if flow $i$ uses link $l$, and 0 otherwise. Let $L(i)$ be the set of links in the path of flow $i$, and $I(l)$ be the set of flows that use link $l$

$$
L(i):=\left\{l \mid R_{l i}=1\right\} \quad I(l):=\left\{i \mid R_{l i}=1\right\} .
$$

Note that $l \in L(i) \Leftrightarrow i \in I(l)$. 
We will present a continuous-time fluid model of XCP. For flows $i$, define the following variables:

- $w_{i}(t)$ : window size at time $t$, in packets;

- $\tau_{i}$ : round-trip propagation (and fixed processing) delay;

- $T_{i}(t)$ : round-trip time (RTT) at time $t$;

- $x_{i}(t):=w_{i}(t) / T_{i}(t)$ : flow rate at time $t$.

For links $l$, define the following variables:

- $c_{l}$ : capacity, in packets/s.

- $b_{l}(t)$ : backlog at time $t$, in packets.

- $y_{l}(t):=\sum_{i} R_{l i} x_{i}(t)$ : aggregate input rate at link $l$ at time $t$. In equilibrium, we sometimes write $y_{l}(x)$ to emphasize the dependence on equilibrium rates $x$.

An XCP link divides time into control intervals of duration $d_{l}$, which is also used as a time scaling parameter. Nominally, $d_{l}$ is the mean RTT of all the flows at link $l$ [9]. We assume $d_{l}$ to be constant in time, which is reasonable as we will show that there is no queueing delay in equilibrium.

To simplify notation, we assume all packets have size 1 unit. We use "flow" and "source" interchangeably.

\section{A. XCP Description}

We now summarize the XCP algorithm. See [9] for a detailed description. The equilibrium is independent of feedback delay, and hence such delay is not modeled.

For each packet, XCP generates a feedback signal prescribing a change in window size. Let $\tilde{H}_{l k}(t)$ be the feedback generated by link $l$ for packet $k$ at time $t$. The acknowledgment for packet $k$ received by its source contains in its header the smallest feedback $\min _{l} \tilde{H}_{l k}(t)$ generated by links along its path. The source adds this quantity to its current window size. ${ }^{1}$ We now describe how to compute the feedback.

Let

$$
\phi_{l}(t)=\alpha d_{l}\left(c_{l}-y_{l}(t)\right)-\beta b_{l}(t)
$$

where $\alpha, \beta>0$ are constants, $c_{l}$ is the link capacity, $y_{l}(t)$ is the aggregate input rate, and $b_{l}(t)$ is the backlog at time $t$. Let $\phi_{l}^{+}(t)=\max \left(\phi_{l}(t), 0\right)$ and $\phi_{l}^{-}(t)=\max \left(-\phi_{l}(t), 0\right)$. The feedback on the $k$ th packet at link $l$ is

$$
\tilde{H}_{l k}(t)=\tilde{p}_{l k}(t)-\tilde{n}_{l k}(t)
$$

where $\tilde{p}_{l k}(t)$ and $\tilde{n}_{l k}(t)$ are the increase and decrease components, respectively

$$
\begin{aligned}
& \tilde{p}_{l k}(t)=\left(h_{l}(t)+\phi_{l}^{+}(t)\right) \frac{\tilde{T}_{k}(t)}{d_{l}} \frac{\tilde{T}_{k}(t) / \tilde{w}_{k}(t)}{\sum_{j=1}^{K_{l}(t)} \tilde{T}_{j}(t) / \tilde{w}_{j}(t)} \\
& \tilde{n}_{l k}(t)=\left(h_{l}(t)+\phi_{l}^{-}(t)\right) \frac{\tilde{T}_{k}(t)}{d_{l}} \frac{1}{K_{l}(t)}
\end{aligned}
$$

where $\tilde{T}_{k}(t)$ and $\tilde{w}_{k}(t)$ are the round-trip time and window size, respectively, of the flow that transmitted packet $k$, and $K_{l}(t)$ is the total number of packets seen by link $l$ over the time interval $\left(t-d_{l}, t\right]$. Here

$$
h_{l}(t)=\max \left(0, \gamma d_{l} y_{l}(t)-\left|\phi_{l}(t)\right|\right)
$$

\footnotetext{
${ }^{1}$ In practice, the window size has a lower bound of 1 packet, but for notational simplicity, we ignore this.
}

is a "traffic shuffling" term with $\gamma \geq 0$ a constant. (Note that we are using the definition of $\gamma$ from the Appendix of [9], which differs by a factor of $d_{l}$ from that used in the corresponding equation in [9].)

\section{B. Dynamic Model}

We now translate the per-packet feedback $\tilde{H}_{l k}(t)$ into per-flow feedback. Let $H_{l i}(t)$ be the feedback generated by link $l$ for flow $i$ at time $t$. In general, a quantity with a tilde $\left(^{\sim}\right)$ pertains to a packet while the corresponding variable without a tilde pertains to a flow.

Substituting $\tilde{x}_{k}(t)=\tilde{w}_{k}(t) / \tilde{T}_{k}(t)$ in (1) gives

$$
\tilde{p}_{l k}(t)=\frac{\tilde{T}_{k}(t)}{\tilde{x}_{k}(t)} \frac{h_{l}(t)+\phi_{l}^{+}(t)}{d_{l} \sum_{i=j}^{K_{l}(t)} 1 / \tilde{x}_{j}(t)} .
$$

Recall $K_{l}(t)$ is the total number of packets arriving at link $l$ in period $\left(t-d_{l}, t\right]$. For simplicity, we assume that

$$
K_{l}(t)=y_{l}(t) d_{l}=d_{l} \sum_{i} R_{l i} x_{i}(t)
$$

Of these packets, we assume that $R_{l i} x_{i}(t) d_{l}$ packets are from flow $i$. Hence

$$
\sum_{j=1}^{K_{l}(t)} \frac{1}{\tilde{x}_{j}(t)}=\sum_{i=1}^{N} R_{l i} x_{i}(t) d_{l} \cdot \frac{1}{x_{i}(t)}=N_{l} d_{l}
$$

Thus, the feedback (3) yields feedback to flow $i$

$$
p_{l i}(t)=\frac{T_{i}(t)}{d_{l}^{2}} \frac{h_{l}(t)+\phi_{l}^{+}(t)}{N_{l} x_{i}(t)}
$$

per packet. Using $K_{l}(t)=y_{l}(t) d_{l}$ again, the per-packet feedback (2) becomes

$$
n_{l i}(t)=\frac{T_{i}(t)}{d_{l}^{2}} \frac{h_{l}(t)+\phi_{l}^{-}(t)}{y_{l}(t)} .
$$

The feedback per packet to flow $i$ from link $l$ is then

$$
H_{l i}(t)=\frac{T_{i}(t)}{d_{l}^{2}}\left(\frac{h_{l}(t)+\phi_{l}^{+}(t)}{N_{l} x_{i}(t)}-\frac{h_{l}(t)+\phi_{l}^{-}(t)}{y_{l}(t)}\right) .
$$

If flow $i$ does not use link $l$, then set $H_{l i}(t)=\infty$.

Let $H_{i}(t)=\min _{l \in L(i)} H_{l i}(t)$ be the minimum feedback along $i$ 's path. Since source $i$ receives $x_{i}(t)$ feedback packets per unit time (assuming every packet carries control information and is acknowledged), its window evolves according to

$$
\dot{w}_{i}(t)=x_{i}(t) \cdot H_{i}(t) \text {. }
$$

Substituting $x_{i}(t)=w_{i}(t) / T_{i}(t)$, we have

$$
\dot{w}_{i}(t)=w_{i}(t) \min _{l \in L(i)}\left(\frac{h_{l}(t)+\phi_{l}^{+}(t)}{d_{l}^{2} N_{l} x_{i}(t)}-\frac{h_{l}(t)+\phi_{l}^{-}(t)}{d_{l}^{2} y_{l}(t)}\right) .
$$

Remark: The pseudo code in [9] contains additional "residual" terms. These terms, modeled in [20], have no effect on the equilibrium if the average rate of flows bottlenecked 
at upstream links is significant. Otherwise, the resulting link utilization is slightly increased (by around 4\% in Scenario 1 of Section V). Since these terms hardly affect the equilibrium, we ignore them in the analysis in this paper.

In summary, an XCP network is described by the following set of equations:

$$
\begin{aligned}
\dot{w}_{i}(t) & =w_{i}(t) \min _{l \in L(i)} F_{l i}(t) \\
\dot{b}_{l}(t) & = \begin{cases}y_{l}(t)-c_{l}, & \text { if } b_{l}(t)>0 \\
\max \left(y_{l}(t)-c_{l}, 0\right), & \text { if } b_{l}(t)=0\end{cases}
\end{aligned}
$$

where

$$
\begin{aligned}
F_{l i}(t) & =\frac{1}{d_{l}^{2}}\left(\frac{h_{l}(t)+\phi_{l}^{+}(t)}{N_{l} x_{i}(t)}-\frac{h_{l}(t)+\phi_{l}^{-}(t)}{y_{l}(t)}\right) \\
\phi_{l}(t) & =\alpha d_{l}\left(c_{l}-y_{l}(t)\right)-\beta b_{l}(t) \\
h_{l}(t) & =\max \left(\gamma d_{l} y_{l}(t)-\left|\phi_{l}(t)\right|, 0\right) \\
x_{i}(t) & =\frac{w_{i}(t)}{T_{i}(t)} \\
y_{l}(t) & =\sum_{i} R_{l i} x_{i}(t) \\
T_{i}(t) & =\tau_{i}+\sum_{l} R_{l i} \frac{b_{l}(t)}{c_{l}} .
\end{aligned}
$$

Here, $\alpha>0, \beta \geq 0, \gamma \geq 0$ are constants, and $\phi_{l}^{+}(t)=$ $\max \left(\phi_{l}(t), 0\right), \phi_{l}^{-}(t)=\max \left(-\phi_{l}(t), 0\right)$. Standard XCP uses $\alpha=0.4, \beta=0.226$ and $\gamma=0.1$. We will study the behavior of the general model, which includes this as a special case. As we will see below, the qualitative properties, such as existence and uniqueness of equilibrium rates, do not depend on specific values of these parameters (as long as $\gamma>0$ ).

\section{EQUILIBRIUM RATES}

This section characterizes the equilibrium of XCP and describes an algorithm to compute it; the next considers the implications of these results on utilization and fairness.

Equations (4)-(5) describe the evolution of the window vector $w(t)=\left(w_{i}(t)\right.$, for all $\left.i\right)$ and the backlog vector $b(t)=$ $\left(b_{l}(t)\right.$, for all $\left.l\right)$. A pair of rate and backlog vectors $(x, b)$, with window vector $w$ given by $w_{i}=x_{i}\left(\tau_{i}+\sum_{l} R_{l i} b_{l} / c_{l}\right)$, is said to be in equilibrium if both $\dot{w}(t)=0$ and $\dot{b}(t)=0$. To avoid technicalities, we only consider cases with $w_{i}>0$; under a minor reformulation, it can be shown that $w_{i}=0$ in equilibrium only if $\gamma=0$, and all theorems and lemmas still hold.

We start by defining a bottleneck link and other notation for $\mathrm{XCP}$ equilibrium. In general, quantities without $t$ dependence denote equilibrium quantities, e.g., $w_{i}, T_{i}, x_{i}, F_{l i}$.

Definition 1: A link $l$ is said to be a bottleneck for source $i$ with respect to (w.r.t.) $x$ if $F_{l i}$ is minimum among all the links that $i$ uses, i.e., $F_{l i}=\min _{m \in L(i)} F_{m i}$. In this case, source $i$ is said to be bottlenecked at link $l$ w.r.t. $x$.

By definition, every source $i$ has a bottleneck. Lemma 1 below implies that $F_{l i}=0$ in equilibrium at bottleneck $l$.
We distinguish between links that are bottlenecks and those that are not. Let $L_{1}(i)$ be the set of links that are bottlenecks for source $i$ w.r.t. a given equilibrium rate $x$

$$
L_{1}(i):=\left\{l \in L(i) \mid F_{l i}=\min _{m \in L(i)} F_{m i}\right\}
$$

and $L_{0}(i):=L(i) \backslash L_{1}(i)$ be the set of links in $i$ 's path that are not bottlenecks for source $i$ w.r.t. $x$. We also distinguish between sources that are bottleneck locally and those that are not. Let $I_{1}(l)$ be the set of sources bottlenecked at link $l$ w.r.t. a given equilibrium rate $x$

$$
I_{1}(l):=\left\{i \in I(l) \mid F_{l i}=\min _{m \in L(i)} F_{m i}\right\}
$$

and $I_{0}(l):=I(l) \backslash I_{1}(l)$ be the set of sources bottlenecked elsewhere. Let $N_{l}:=|I(l)|$ be the number of sources at link $l, N_{l 0}:=\left|I_{0}(l)\right|$, and $N_{l 1}:=\left|I_{1}(l)\right|$. Let $\rho_{l}:=N_{l 0} / N_{l}$ be the fraction of flows through link $l$ which are not bottlenecked at link $l$, and $\sigma_{l}:=y_{l 0} / c_{l}$ be the fraction of the link's capacity consumed by such flows. Note that while $L(i), I(l)$, and $N_{l}$ depend only on $R$ the routing matrix, $L_{1}(i), L_{0}(i), I_{1}(l), I_{0}(l)$, $N_{l 0}, N_{l 1}, \rho_{l}$ and $\sigma_{l}$ depend also on the equilibrium rate $x$ through $F_{l i}$.

From (4) and the definition of $I_{0}(l)$, we have the following.

Lemma 1: The rate and backlog vector $(x, b)$ is in equilibrium if and only if

1) for all $l, y_{l} \leq c_{l}$ with equality if $b_{l}>0$, and

2) for all $i, \min _{l \in L(i)} F_{l i}=0$.

Moreover,

3) if $i \in I_{0}(l)$ and $j \in I_{1}(l)$ then $F_{l i}>0$ and $F_{l j}=0$;

4) if $I_{1}(l) \neq \emptyset$ then $h_{l}=0$ implies $\phi_{l}=0$.

Proof: Parts 1 to 3 are immediate. To see part 4, note that $h_{l}=0$ implies

$$
F_{l i}=\frac{1}{d_{l}^{2}}\left(\frac{\phi_{l}^{+}}{N_{l} x_{i}}-\frac{\phi_{l}^{-}}{y_{l}}\right)
$$

By part 2, $F_{l i}=0$ for all $i \in I_{1}(l)$. Since at most one of $\phi_{l}^{+}$and $\phi_{l}^{-}$is nonzero, $\phi_{l}^{+}=\phi_{l}^{-}=0$, whence $\phi_{l}=0$.

\section{A. The Need for Bandwidth Shuffling}

Without bandwidth shuffling, XCP would have $\gamma=0$, giving $h_{l}(t)=0$ for all $l$ and $t$, and $h_{l}=0$ in equilibrium.

Theorem 1: Suppose $\gamma=0$. Then, $(x, b)$ with $x_{i}=w_{i} / \tau_{i}$ is an equilibrium if and only if

1) for all $l, y_{l} \leq c_{l}$ and $b_{l}=0$, and

2) for all $i$, there exists $l \in L(i)$ with $y_{l}=c_{l}$.

Proof: The first condition in the theorem implies that for all $l, \phi_{l} \geq 0$. Combined with $h_{l}=0$, this implies $F_{l i} \geq 0$ for all $i$. The second condition then implies that for all $i, \min _{l \in L(i)} F_{l i}=$ 0 . Hence, the conditions in the theorem are sufficient, by (5b) and the first half of Lemma 1.

For necessity, there are two cases. If $I_{1}(l) \neq \emptyset$ then $\phi_{l}=0$ by Lemma 1 part 4, and (4b) implies $y_{l}=c_{l}$ and $b_{l}=0$, since $y_{l} \leq c_{l}, b_{l} \geq 0$, and $\beta>0$. Otherwise, $l \in \bigcap_{i} L_{0}(i)$ and $F_{l i}>0$ by definition of $L_{0}(i)$. This implies $\phi_{l}^{+}>0$, whence $y_{l}<c_{l}, b_{l}=0$ in equilibrium. 
Remark: Without bandwidth shuffling, any (possibly unfair) boundary point of the set $\{x \mid R x \leq c\}$ would be an equilibrium. This is why XCP uses $\gamma>0$ [9].

The rest of the paper considers the more complicated case of $\gamma>0$.

\section{B. $\gamma>0$ Case: Definitions and Preliminaries}

This subsection provides a conceptually simple characterization which is used in the next subsection to prove the existence and uniqueness of XCP equilibrium. Following that, we provide an iterative algorithm to compute this equilibrium.

From (4)-(5) and Lemma 1, $(x, b)$ is an XCP equilibrium if and only if it satisfies conditions 1 and 2 of Lemma 1.

By (5a), condition 2 becomes: for all $i$, for all $l \in L(i)$

$$
x_{i} \leq \frac{y_{l}}{N_{l}} \frac{h_{l}+\phi_{l}^{+}}{h_{l}+\phi_{l}^{-}}=: r^{l}
$$

with equality for some $l \in L(i)$. Hence for links $l$ with $I_{1}(l) \neq$ $\emptyset$, all flows $i \in I_{1}(l)$ that are bottlenecked at link $l$ must have the common rate $r^{l}$. This has important implications as we will see below.

Several of the results will use the following technical lemma, which is proved in Appendix A.

Lemma 2: For all $l$

1) $x_{i}<x_{j}=r^{l}$ if $i \in I_{0}(l)$ and $j \in I_{1}(l)$;

2) $\sigma_{l}<\rho_{l}$ if $I_{0}(l) \neq \emptyset$;

3) $r^{l} \geq y_{l} / N_{l}$ with equality if and only if $I_{0}(l)=\emptyset$;

4) $h_{l}>0$ if $I_{1}(l) \neq \emptyset ; 2$

5) $y_{l} / c_{l} \geq \sigma_{l}$ with equality if and only if $I_{1}(l)=\emptyset$.

Unlike in the $\gamma=0$ case, we characterize the equilibrium backlogs and rates separately. The following result says that the equilibrium queue under XCP is zero. This originates from the definition of $\phi_{l}$ in (5b), which is nonnegative in equilibrium. The same property is used in REM [22] to drive the queue to zero, or more generally, to a target value.

Theorem 2: In equilibrium, $b_{l}=0$ and $\phi_{l} \geq 0$ for all $l$.

Proof: Links can be of three types: a) $I_{1}(l) \neq \emptyset, I_{0}(l)=\emptyset$; b) $I_{1}(l)=\emptyset, I_{0}(l) \neq \emptyset$; and c) $I_{1}(l) \neq \emptyset, I_{0}(l) \neq \emptyset$. Each of these will be considered in turn.

Type a) links $l$ are bottlenecks for all flows passing through them, i.e., (6) holds with equality for all $i \in I(l)$. Since all flows have common rate $r^{l}, y_{l}=N_{l} r^{l}$, whence equality in (6) implies $\phi_{l}^{+}=\phi_{l}^{-}$. Thus, $\phi_{l}=0$, and (5b) implies $y_{l}=c_{l}$ and $b_{l}=0$, i.e., they share the link capacity fully and equally, with no queueing delay.

Type b) links are not bottlenecks for any of the flows they carry. Hence, for all $i \in I(l)$,

$$
x_{i}<\frac{y_{l}}{N_{l}} \frac{h_{l}+\phi_{l}^{+}}{h_{l}+\phi_{l}^{-}}
$$

Multiplying both sides by $R_{l i}$ and summing over $i$, we have

$$
y_{l}<\frac{y_{l}}{N_{l}} \frac{h_{l}+\phi_{l}^{+}}{h_{l}+\phi_{l}^{-}} \cdot \sum_{i} R_{l i}
$$

\footnotetext{
${ }^{2} h_{l}>0$ implies $y_{l} / c_{l}>\alpha /(\alpha+\gamma)$. Theorem 6 provides a tighter lower bound.
}

Hence

$$
\frac{h_{l}+\phi_{l}^{+}}{h_{l}+\phi_{l}^{-}}>1
$$

Since both numerator and denominator are positive, $\phi_{l}^{+}>\phi_{l}^{-}$. This implies $\phi_{l}>0$ whence $y_{l}<c_{l}$ and $b_{l}=0$.

Type c) links are bottleneck links for some but not all of the flows using them. From (6), we have

$$
\frac{h_{l}+\phi_{l}^{+}}{h_{l}+\phi_{l}^{-}}=\frac{r^{l}}{y_{l} / N_{l}}>1
$$

where the inequality follows from Lemma 2 . As for type b) links, this implies $\phi_{l}>0, y_{l}<c_{l}$ and $b_{l}=0$.

We next characterize XCP's equilibrium rates. Let

$$
g_{l}(x):=\frac{\gamma y_{l}^{2}}{N_{l}\left[(\gamma+\alpha) y_{l}-\alpha c_{l}\right]}
$$

where $y_{l}=\sum_{i} R_{l i} x_{i}$. Since $g_{l}(x)$ depends on $x$ only through $y_{l}$, we will abuse notation and also write $g_{l}\left(y_{l}\right)$ or $g_{l}\left(y_{l}(x)\right)$. Define the feasible set of source rates $x$ to be

$$
X_{0}:=\left\{x \in \mathbb{R}_{+}^{N} \mid g_{l}\left(y_{l}\right)<0 \text { or } x_{i} \leq g_{l}\left(y_{l}\right), \forall l, i \in I(l)\right\}
$$

where $\mathbb{R}_{+}$denotes the set of nonnegative real numbers. We will later show that the $\mathrm{XCP}$ equilibrium must be in $X_{0}$. Note that $x \in X_{0}$ implies

$$
R x \leq c .
$$

To see this, multiply both sides of the inequalities in (7) by $R_{l i}$ and sum over $i$ to get

$$
\frac{\gamma y_{l}^{2}}{(\gamma+\alpha) y_{l}-\alpha c_{l}} \notin\left[0, \sum_{i} R_{l i} x_{i}\right)=\left[0, y_{l}\right)
$$

whence $y_{l} \leq c_{l}$. The converse may not be true, i.e., $X_{0}$ may be a strict subset of $\{x \mid R x \leq c\}$.

Intuitively, $g_{l}$ exceeds the equal-share rate $y_{l} / N_{l}$ by a factor that increases as the link underutilization increases and as the bandwidth shuffling decreases. It arises since the link tries to shuffle bandwidth from flows it controls to ones it does not. Thus, $X_{0}$ restricts the utilization of links which are not bottlenecks for all flows passing through them.

Our main result is to prove the existence and uniqueness of $\mathrm{XCP}$ equilibrium in a general network, and that this equilibrium solves a constrained max-min fairness problem.

Definition 2: A rate vector $x^{*} \in X_{0}$ is constrained max-min fair if for any other feasible $x \in X_{0}, x_{i}>x_{i}^{*}$ implies that there is a $j$ with $x_{j}<x_{j}^{*}$ and $x_{j}^{*} \leq x_{i}^{*}$.

Intuitively, a constrained max-min fair vector $x^{*}$ is such that it is not possible to increase a component $x_{i}^{*}$ without reducing another smaller or equal component $x_{j}^{*}$. This differs from standard max-min fairness only in that the feasible set $X_{0}$ is a subset of $\{x \mid R x \leq c\}$ [23]. This restriction has important ramifications, as we will see in the next section.

We will prove constructively that the unique XCP equilibrium is constrained max-min fair by identifying it with the solution of 
a hierarchy of optimization problems over the feasible set $X_{0}$ : it maximizes the smallest source rates in $X_{0}$, and then maximizes the second smallest rates over all rates that solve the first problem, and so on. These problems are defined inductively, following the idea of [24].

Let $L_{0}=\emptyset$ and $I_{0}=\emptyset$. The sets $\left(L_{0}, I_{0}, X_{0}\right)$ define the first problem $\mathbf{P}_{1}$, whose solution is described by the sets $\left(L_{1}, I_{1}, X_{1}\right)$. These sets in turn define the second problem $\mathbf{P}_{2}$, and so on. To simplify notation, let

$$
\bar{L}_{n}:=\bigcup_{m \leq n} L_{m} \quad \bar{I}_{n}:=\bigcup_{m \leq n} I_{m} .
$$

Given sets $\left(X_{0}, L_{0}, I_{0}\right), \ldots,\left(X_{n-1}, L_{n-1}, I_{n-1}\right)$, if $\bar{I}_{n-1}$ contains all flows, then we stop. Otherwise, we define problem $\mathbf{P}_{n}$ and its solution $L_{n}, I_{n}, X_{n}, n \geq 1$, as follows:

$$
\mathbf{P}_{n}: \max _{x \in X_{n-1}} \min _{i \notin \bar{I}_{n-1}} x_{i}
$$

Let

$$
\begin{aligned}
r_{n} & :=\min _{l \notin \bar{L}_{n-1}} \max _{x \in X_{n-1}} g_{l}(x) \\
L_{n} & :=\{\operatorname{minimizing} l \text { in }(9)\} \\
I_{n} & :=\bigcup_{l \in L_{n}} I(l) \backslash \bar{I}_{n-1} \\
X_{n} & :=\left\{x \in X_{n-1} \mid x_{i}\left\{\begin{array}{ll}
=r_{n}, & \forall i \in I_{n} \\
>r_{n}, & \forall i \notin \bar{I}_{n}
\end{array}\right\} .\right.
\end{aligned}
$$

A few important properties are immediate from these definitions. First, the rates $r_{n}$ are monotonic

$$
\min _{l} \frac{c_{l}}{N_{l}}=r_{1}<r_{2}<\cdots<r_{n} .
$$

Second, $L_{n}$ and $I_{n}$ are nonempty; moreover, they are disjoint from $\bar{L}_{n-1}$ and $\bar{I}_{n-1}$, respectively. Hence, $\bar{I}_{n}$ will eventually contain all the flows and there are only a finite number of problems $\mathbf{P}_{n}$. Finally, $X_{n}$ are strictly nested

$$
X_{0} \supsetneq X_{1} \supsetneq \cdots \supsetneq X_{n}
$$

Indeed it will become clear that $X_{n}$ is exactly the set of solutions to problem $\mathbf{P}_{n}$, i.e., $X_{1}$ is the set of feasible rates $x \in X_{0}$ whose smallest rates are maximized, $X_{2}$ is a subset of $X_{1}$ whose second smallest rates are also maximized, and so on. We prove below that if $\mathbf{P}_{n *}$ is the last problem, then $X_{n^{*}}$ is a singleton that solves all problems $\mathbf{P}_{1}, \ldots, \mathbf{P}_{n^{*}}$.

To contrast XCP equilibrium with the standard max-min fair allocation, we derive a "bottleneck" characterization that is analogous to that for max-min fairness; see the beginning of Section IV.

Lemma 3: Let $x$ be an XCP equilibrium rate vector. Link $l$ is a bottleneck for source $i \in I(l)$ w.r.t. $x$ if and only if

1) $x_{i}=g_{l}(x)$, and

2) $x_{i} \geq x_{j}$ for all $j \in I(l)$.

Proof: Suppose link $l$ is a bottleneck link for source $i$ w.r.t. equilibrium $x$. Then, Lemma 1-2 implies that $F_{l i}=0$, i.e., equality holds in (6). Since $\phi_{l} \geq 0$ by Theorem 2 and $h_{l}>0$ by Lemma $2,(5 \mathrm{c})$ becomes $h_{l}=\gamma d_{l} y_{l}-\phi_{l}$. Thus from (6)

$$
x_{i}=r^{l}=\frac{y_{l}}{N_{l}} \frac{\gamma d_{l} y_{l}}{\gamma d_{l} y_{l}-\phi_{l}}=g_{l}(x)
$$

proving the first condition. Condition (6) then implies the second condition.

Conversely, suppose the two conditions are satisfied. If $h_{l}=$ 0 , then $F_{l i}=0$ from (5a) and Lemma 1-4. Lemma 1-2 then implies $F_{l i}$ is the minimum among links in source $i$ 's path, i.e., link $l$ is a bottleneck. On the other hand, if $h_{l}>0$, then, as above, $\phi_{l} \geq 0$ and $h_{l}=\gamma d_{l} y_{l}-\phi_{l}$. Then, $x_{i}=g_{l}(x)$ is equivalent to $F_{l i}=0$, proving that $l$ is a bottleneck.

Motivated by this lemma, we call link $l$ a nonbottleneck w.r.t. $x$ if either $g_{l}(x)<0$ or $x_{i}<g_{l}(x)$ for all $i \in I(l)$.

\section{C. $\gamma>0$ Case: Main Result}

We are now ready to state the main result.

Theorem 3: The problems $\mathbf{P}_{n}$ are well-defined and have a unique solution. Moreover, the following are equivalent:

1) $x^{*}$ is an XCP equilibrium.

2) $x^{*}$ is the unique rate vector that solves all the problems $\mathbf{P}_{n}$.

3) $x^{*}$ is constrained max-min fair.

4) $x^{*} \in X_{0}$ and every flow has a bottleneck w.r.t. $x^{*}$, i.e., for all $i$, there is an $l \in L(i)$ such that $x_{i}^{*}=g_{l}\left(x^{*}\right)$ and $x_{i}^{*} \geq x_{j}^{*}$ for all $j \in I(l)$.

The remainder of this subsection is a proof of this theorem. It will use lemmas, whose proofs are relegated to Appendix B. We start with a simple observation that greatly simplifies the solution of $\mathbf{P}_{n}$.

Lemma 4: Suppose $X_{n}$ is nonempty. The maximization in (9) can be taken over $x \in X_{n}$ that have equal $x_{i}$ for $i \notin \bar{I}_{n}$.

In view of Lemma 4 , we can replace $X_{n}$ in (12), for $n \geq 1$, by their subsets

$$
\hat{X}_{n}:=\left\{x \in X_{n-1} \mid \exists \epsilon>0: x_{i}=\left\{\begin{array}{cl}
r_{n}, & i \in I_{n} \\
r_{n}+\epsilon, & i \notin \bar{I}_{n}
\end{array}\right\}\right.
$$

and use them instead of $X_{n-1}$ in computing $r_{n}$ :

$$
r_{n}:=\min _{l \notin \bar{L}_{n-1}} \max _{x \in \hat{X}_{n-1}} g_{l}(x) .
$$

This greatly reduces the complexity of (9) from maximizing over $n$-vectors $x \in X_{n}$ to over a scalar $\epsilon>0$.

Denote an $x \in \hat{X}_{n}$ by $x(\epsilon ; n)$, with

$$
x_{i}(\epsilon ; n)= \begin{cases}r_{m}, & i \in I_{m}, m \leq n \\ r_{n}+\epsilon, & i \notin \bar{I}_{n}\end{cases}
$$

and let $x(0 ; n):=\lim _{\epsilon \rightarrow 0} x(\epsilon ; n)$. Note that $x(0 ; n), n \geq 1$, is not in $X_{n}$ according to definition (12), though it is in $X_{0}$. We will see in Lemma 6 below that $x(0, n)$ plays an important role in the proof of Theorem 3 . The vector $x(\epsilon ; n)$ induces link flows

$$
\begin{aligned}
y_{l}(\epsilon ; n) & =\sum_{i} R_{l i} x_{i}(\epsilon ; n) \\
& =\sum_{m=1}^{n} r_{m} \sum_{i \in I_{m}} R_{l i}+r_{n} \sum_{i \notin \bar{I}_{n}} R_{l i}+\epsilon \sum_{i \notin \bar{I}_{n}} R_{l i} .
\end{aligned}
$$

This motivates the following main technical lemma. 
Lemma 5: Given any scalars $z \geq 0, \eta>0$, and $s \geq 0$, define

$$
\begin{aligned}
\hat{g}_{l}(\epsilon) & :=\hat{g}_{l}(\epsilon ; z, \eta):=g_{l}(z+\eta \epsilon) \\
& =\frac{\gamma(z+\eta \epsilon)^{2}}{N_{l}\left((\gamma+\alpha)(z+\eta \epsilon)-\alpha c_{l}\right)}
\end{aligned}
$$

for some $N_{l} \geq 1, \alpha>0, \gamma>0$ and $c_{l}>0$.

1) If either $\hat{g}_{l}(0)<0$ or $s \leq \hat{g}_{l}(0)$, then there exists a unique $\epsilon_{l} \geq 0$ such that $s+\eta \epsilon_{l}=\hat{g}_{l}\left(\epsilon_{l}\right)$, where $\epsilon_{l}=0$ if and only if $s=\hat{g}_{l}(0)$.

2) Moreover, over $\left\{\epsilon \mid \hat{g}_{l}(\epsilon)>0\right\}, s+\eta \epsilon \leq \hat{g}_{l}(\epsilon)$ if and only if $\epsilon \leq \epsilon_{l}$.

For later reference, we will denote the mapping from $(z, \eta, s)$ to the unique $\epsilon_{l}$ in Lemma 5 by

$$
G_{l}(z, \eta, s)=\epsilon_{l} .
$$

This function is used in the rest of the proof.

Lemma 5 implies that if link $l$ is a bottleneck for some source $i$ with respect to an $x \in X_{0}$, then the rate of source $i$ cannot be increased without violating the feasibility constraint in (7). For instance, let $n \geq 1$ be such that $l \in L_{n}$. Setting $z=y_{l}(0 ; n-$ $1), s=r_{n-1}$ and $\eta=\sum_{i \notin \bar{I}_{n-1}} R_{l i}$ gives $\epsilon_{l}=r_{n}-r_{n-1}$ and $l$ is a bottleneck for all $i \in I_{n}$ w.r.t. $x\left(\epsilon_{l} ; n-1\right)$. Lemma 5-2 then implies that rates greater than $r_{n}$ are infeasible at link $l$.

The next lemma implies that all links $l \in L_{n}$ are bottlenecks w.r.t. all $x \in \hat{X}_{n}$, and all links $l \notin \bar{L}_{n}$ are nonbottlenecks w.r.t. $x(0 ; n)$. In particular, this implies that $X_{n}$ are nonempty.

Lemma 6: For each $n \geq 1$,

1) if $l \in L_{n}$, then $x_{i}=\bar{g}_{l}(x)$ for all $i \in I_{n}$ w.r.t. all $x \in \hat{X}_{n}$.

2) if $l \notin \bar{L}_{n}$, then either $g_{l}(x(0 ; n))<0$ or $x_{i}(0 ; n)<$ $g_{l}(x(0 ; n))$ for all $i \in I(l)$.

Lemmas 5 and 6 suggest the following abstract algorithm to compute the solution of problems $\mathbf{P}_{n}$. An analogous concrete algorithm to find the XCP equilibrium will be given in the Subsection III-D. At the start of iteration $n+1$, all links $l \in L_{m}, m \leq n$, are bottlenecks for some sources $i \in I_{m}$ w.r.t. all $x \in X_{m}$. A source $i \in \bar{I}_{n}$ passes through at least one bottleneck $l \in \bar{L}_{n}$, and hence its rate cannot be raised further without violating the constraint in (7). All links $l \notin \bar{L}_{n}$ are nonbottleneck links w.r.t. $x(0 ; n)$ defined in (16). Sources $i \notin \bar{I}_{n}$ pass through only these nonbottlenecks, and hence their rates can be increased further, starting from $r_{n}$. At each nonbottleneck link $l$, nonbottlenecked sources $i \notin \bar{L}_{n}$ can raise their rates to (19), (16), and (17)

$$
r_{n}+G_{l}\left(\sum_{m=1}^{n} r_{m} \sum_{i \in I_{m}} R_{l i}+r_{n} \sum_{i \notin \bar{I}_{n}} R_{l i}, \sum_{i \notin \bar{I}_{n}} R_{l i}, r_{n}\right)
$$

to make $l$ a bottleneck. The smallest of these rates, smallest over $l \notin \bar{L}_{n}$, is $r_{n+1}$. It is assigned to all previously nonbottlenecked sources going through the new bottleneck links and is the optimal objective value for problem $\mathbf{P}_{n}$. These new bottleneck links are collected into $L_{n+1}$, the newly bottlenecked sources into $I_{n+1}$ and their rates into $\hat{X}_{n+1}\left(\right.$ or $\left.X_{n+1}\right)$. The other nonminimizing links remain nonbottleneck w.r.t. the new rates $x \in \hat{X}_{n+1}$, and the cycle repeats, until all sources are assigned their bottleneck rates.

The solution of each problem $\mathbf{P}_{n}$ fixes the components $x_{i}, i \in I_{n}$, to be rate $r_{n}$, until all components have been assigned. Hence, if $\mathbf{P}_{n^{*}}$ is the last problem, then $X_{n^{*}}=\left\{x^{*}\right\}$ is a singleton.

The above discussion is summarized in the following lemma, which justifies Theorem 3.

Lemma 7: The problems $\mathbf{P}_{n}$ are well defined. $X_{n}$ is exactly the set of solutions to problem $\mathbf{P}_{n}$. There is a unique solution to the hierarchy of problems.

We now prove Theorem 3.

Proof (Theorem 3): Lemma 7 implies that $\mathbf{P}_{n}$ are well defined and have a unique solution. It is clear that characterizations 2 and 3 are equivalent, i.e., $x^{*}$ is the unique solution to the hierarchy of problems $\mathbf{P}_{n}$ if and only if it is constrained max-min fair. We will first prove the equivalence of characterizations 3 and 4 , and then that of 1 and 4 . We will use the equivalent definition of bottleneck links in Lemma 3 .

Equivalence of characterizations 3 and 4: We will prove that $x^{*}$ is constrained max-min fair if and only if both $x^{*} \in X_{0}$ and every flow $i$ has a bottleneck link w.r.t. $x^{*}$, i.e., for all $i$, there is an $l \in L(i)$ such that $x_{i}^{*}=g_{l}(x)$ and $x_{i}^{*} \geq x_{j}^{*}$ for all $j \in I(l)$. The proof follows the same approach as the corresponding result for standard max-min fairness; see [23]. The difference is in the use of Lemma 5 because of the more complicated feasible set $X_{0}$.

Suppose $x^{*} \in X_{0}$ and every flow $i$ has a bottleneck link w.r.t. $x^{*}$. If $x^{*}$ is not constrained max-min fair, then there exists another $x \in X_{0}$ such that $x_{i}>x_{i}^{*}$ for some $i$, and if $x_{j}^{*} \leq x_{i}^{*}$ then $x_{j} \geq x_{j}^{*}$. We will derive a contradiction. Let $l \in L(i)$ be a bottleneck for $i$ w.r.t. $x^{*}$. Then, $x_{i}^{*} \geq x_{j}^{*}$ for all $j \in I(l)$, and hence $\epsilon_{j}:=x_{j}-x_{j}^{*} \geq 0$ for all $j \in I(l)$, with $\epsilon_{i}>0$. Write the link flow $y_{l}(x)$ due to rates $x$ in terms of $\epsilon_{j}$ and the link flow $y_{l}\left(x^{*}\right)$ due to $x^{*}$

$$
\begin{aligned}
y_{l}(x) & =\sum_{j} R_{l j}\left(x_{j}^{*}+\epsilon_{j}\right) \\
& =y_{l}\left(x^{*}\right)+\sum_{j} R_{l j} \epsilon_{j} .
\end{aligned}
$$

Let the scalar $\epsilon$ be the average $\epsilon_{j}$

$$
\epsilon=\frac{\sum_{j} R_{l j} \epsilon_{j}}{\sum_{j} R_{l j}}>0
$$

Then, the rate vector $\hat{x}$ defined by $\hat{x}_{j}=x_{j}^{*}+\epsilon$ if $j \in I(l)$ and $\hat{x}_{j}=x_{j}^{*}$ otherwise induces the same flow rate at link $l$ as $x$ does

$$
y_{l}(\hat{x})=y_{l}\left(x^{*}\right)+\epsilon \cdot \sum_{j} R_{l j}=y_{l}(x) .
$$

Since $\epsilon \leq \max _{j} \epsilon_{j}$, and $x$ is feasible, we must have, for all $j \in I(l)$

$$
\begin{aligned}
\hat{x}_{j} & \leq \max _{\hat{\jmath} \in I(l)} x_{\hat{\jmath}} \leq g_{l}\left(y_{l}(x)\right) \\
& =g_{l}\left(y_{l}(\hat{x})\right)
\end{aligned}
$$


where the last equality follows from (20). Hence, $\hat{x}$ is also feasible. Since link $l$ is a bottleneck for source $i$ w.r.t. $x^{*}$

$$
x_{i}^{*}=g_{l}\left(y_{l}\left(x^{*}\right)\right)>0
$$

and so applying Lemma 5-1 with $z=y_{l}\left(x^{*}\right), s=x_{i}^{*}$ and $\eta=\sum_{j} R_{l j}$ gives $\epsilon_{l}=0$. Since $\hat{x}_{j}=x_{j}^{*}+\epsilon$ for all $j \in I(l)$, with $\epsilon>\epsilon_{l}$, Lemma 5-2 and (20) imply that

$$
\hat{x}_{i}>g_{l}\left(y_{l}(\hat{x})\right)
$$

contradicting (21). Hence, $x^{*}$ is constrained max-min fair.

Conversely, let $x^{*} \in X_{0}$ be constrained max-min fair. If there is a source $i$ that has no bottleneck link w.r.t. $x^{*}$, then for all $l \in L(i)$, either $g_{l}\left(y_{l}\left(x^{*}\right)\right)<0$ or $x_{i}^{*}<g_{l}\left(y_{l}\left(x^{*}\right)\right)$ for all $i \in I(l)$. Lemma 5 then implies that there exists a unique scalar $\epsilon_{i}>0$, given by

$$
\epsilon_{i}=\min _{l \in L(i)} G_{l}\left(y_{l}\left(x^{*}\right), 1, x_{i}^{*}\right)
$$

such that $x \in X_{0}$, given by $x_{i}=x_{i}^{*}+\epsilon_{i}$ and $x_{j}=x_{j}^{*}$ for $j \neq i$, strictly increases component $i$ without having to reduce other components $j$, contradicting the fact that $x^{*}$ is constrained max-min fair.

Equivalence of characterizations 1 and 4: We will prove that a vector $x^{*} \in \mathbb{R}_{+}^{N}$ is an XCP equilibrium if and only if $x^{*} \in X_{0}$ and every flow $i$ has a bottleneck link w.r.t. $x^{*}$.

The discussion at the beginning of Section III-B shows that $x^{*}$ is an XCP equilibrium if and only if, for all $i,(6)$ holds for all $l \in L(i)$, with equality for some $l \in L(i)$. This, with (14), establishes $x^{*} \in X_{0}$. As observed after Definition 1, every flow has a bottleneck by definition.

To show characterization 4 implies characterization 1, it suffices to show that the characterization in Lemma 3 implies statements 1 and 2 of Lemma 1. The discussion after (7), and setting $b=0$, establishes statement 1 . This shows $\phi_{l}^{-}=0$ for all $l$. If $x_{i} \leq g_{l}(x)$ then $(5 \mathrm{c})$ and (5a) give $F_{l i} \geq 0$, with equality when $x_{i}=g_{l}(x)$. Otherwise, $g_{l}(x)<0$ giving $h=0$ and, by (5a), $F_{l i} \geq 0$.

\section{Algorithm for Computing Equilibrium}

The equilibrium rates of XCP can be found using an algorithm analogous to that of [23] for max-min fairness. However, because the constraint on the link throughput in (6) depends on the aggregate flow rate through $h_{l}$ and $\phi_{l}$, some extra bookkeeping is required.

In [20], an approximation for $y_{l}$ was given in terms of $\sigma_{l}$ and $\rho_{l}$. Here, we use the exact expression from [19].

Theorem 4: The utilization of a bottleneck $l$ satisfies

$$
\frac{y_{l}}{c_{l}}=\frac{\alpha+(\gamma+\alpha) \sigma_{l}+\sqrt{\left(\alpha-(\gamma+\alpha) \sigma_{l}\right)^{2}+4 \alpha \gamma \sigma_{l}\left(1-\rho_{l}\right)}}{2\left(\gamma \rho_{l}+\alpha\right)} .
$$

The rates of all sources $i \in I_{1}(l)$ bottlenecked at $l$ satisfy

$$
r^{l}=\frac{c_{l}}{N_{l}} \times \frac{\left[\Xi_{l}-\gamma \sigma_{l} \rho_{l}\right]+\sqrt{\left[\Xi_{l}+\gamma \sigma_{l} \rho_{l}\right]^{2}-4 \alpha \gamma \sigma_{l}\left(\rho_{l}-\sigma_{l}\right)}}{2\left(1-\rho_{l}\right)\left(\gamma \rho_{l}+\alpha\right)}
$$

where

$$
\Xi_{l}=\left(\gamma \sigma_{l}+\alpha\right)\left(1-\sigma_{l}\right)-\gamma \sigma_{l}\left(\rho_{l}-\sigma_{l}\right) .
$$

Proof: Substituting $r^{l}=\left(y_{l}-y_{l 0}\right) /\left(N_{l}-N_{l 0}\right)$ into (14) and solving the resulting quadratic equation gives

$$
y_{l}=\frac{\alpha c_{l}+(\gamma+\alpha) y_{l 0} \pm \sqrt{\left(\alpha c_{l}+(\gamma+\alpha) y_{l 0}\right)^{2}-K}}{2\left(\gamma N_{l 0} / N_{l}+\alpha\right)}
$$

where $K=4 \alpha c_{l} y_{l 0}\left(\gamma N_{l 0} / N_{l}+\alpha\right)$. By Lemma 8 in Appendix A, only the larger solution of (26) satisfies part 5 of Lemma 2 and is a valid equilibrium. Rearranging the term in the square root gives (23).

To obtain (24), instead substitute $y_{l}=\left(N_{l}-N_{l 0}\right) r^{l}+y_{l 0}$ into (14), giving

$$
A_{l}\left(\frac{r^{l} N_{l}}{c_{l}}\right)^{2}+B_{l} \frac{r^{l} N_{l}}{c_{l}}+C_{l}=0
$$

where

$$
\begin{aligned}
& A_{l}=\left(1-\rho_{l}\right)\left(\gamma \rho_{l}+\alpha\right) \\
& B_{l}=2 \gamma \sigma_{l} \rho_{l}-\gamma \sigma_{l}-\alpha\left(1-\sigma_{l}\right) \\
& C_{l}=-\gamma \sigma_{l}^{2} .
\end{aligned}
$$

Since $y_{l}$ is increasing in $r^{l}$, it is again only the larger root that represents the XCP equilibrium. Thus

$$
\frac{r^{l} N_{l}}{c_{l}}=\frac{\left[\Xi_{l}-\gamma \sigma_{l} \rho_{l}\right]+\sqrt{\left[\Xi_{l}-\gamma \sigma_{l} \rho_{l}\right]^{2}-4 A_{l} C_{l}}}{2\left(1-\rho_{l}\right)\left(\gamma \rho_{l}+\alpha\right)}
$$

where $\Xi_{l}$ is given in (25). Rearranging the expression in the square root gives (24).

Note that the right-hand side of (24) depends on the rate vector $x$ through $\rho_{l}$ and $\sigma_{l}$. Hence, it is not an explicit formula for the throughput of a general flow. However, it says that the common "bottleneck" rate at each link $l$ depends on the rate vector $x$ only through $y_{l 0}$ and $N_{l 0}$ that are bottlenecked elsewhere. These are source rates smaller than the "bottleneck" rate at link $l$ by Lemma 1 . This motivates an algorithm similar to the max-min algorithm of [23] that calculates the throughput $x_{i}$ of each flow in increasing order, without the need for recourse to simulation.

1 Set $\bar{I}_{0} \leftarrow \emptyset, \bar{L}_{0} \leftarrow \emptyset, \sigma_{l}(0) \leftarrow 0, \rho_{l}(0) \leftarrow 0$ for all $l, n \leftarrow 1$

2 repeat

2.1 For each link, $l \notin L_{n-1}$ find $r^{l}(n)$ from (24) using $\sigma_{l}(n-1)$ and $\rho_{l}(n-1)$ from rates already allocated

2.2 Set $r_{n} \leftarrow \min _{j} r^{j}(n)$

2.3 Set $L_{n} \leftarrow\left\{l: r^{l}(n)=r_{n}\right\}$

2.4 foreach $l \in L_{n}$ 2.4.1 Set $r^{l} \leftarrow r_{n}$ 2.4.2 For each flow $i \in I(l) \backslash \bar{I}_{n}$, set $x_{i} \leftarrow r^{l}$ endfor

2.5 Set $I_{n} \leftarrow \bigcup_{j \in L_{n}} I(j) \backslash \bar{I}_{n}$ 


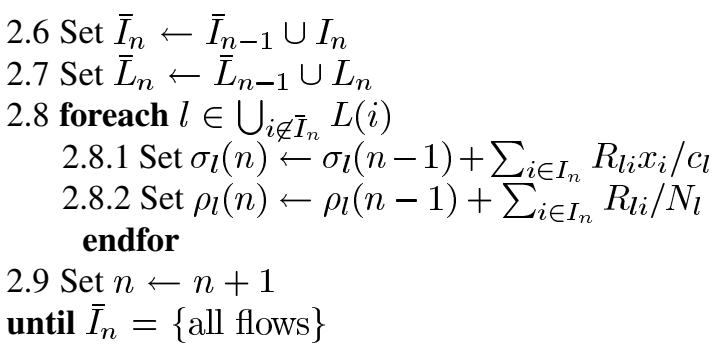

This solves each of the optimization problems, $\mathbf{P}_{n}$, in turn. The key is that, by keeping track of the used capacity of each link, $\sigma_{l}(n)$ and $\rho_{l}(n)$, it can compute the maximization in (9) in closed form. For each $l$, the values $\sigma_{l}(n)$ and $\rho_{l}(n)$ vary during the algorithm. For the algorithm to be correct, they must have the right values when link $l$ is the minimum in step 2.2. This occurs as long as the link rates are allocated in increasing order, as is guaranteed by the following theorem, proved in Appendix C.

Theorem 5: The above algorithm calculates the correct equilibrium rates of $\mathrm{XCP}$.

If $\gamma=0$, then (24) reduces to $r^{l}=\left(c_{l}-y_{l 0}\right) /\left(N_{l}-N_{l 0}\right)$, and hence the algorithm reduces to the algorithm in [23] to compute the max-min fair allocation. This suggests that, given any topology specified by the routing matrix $R$ and link capacity vector $c$, one can choose $\gamma>0$ to be sufficiently small so that the equilibrium of (4) is close to max-min fair. On the other hand, with small $\gamma$, the convergence of individual rates to fairness can be very slow. We will return to this point in the following section.

\section{UTILIZATION AND FAIRNESS}

In this section, we discuss some implications of the results in Section III on link utilization and fairness of the equilibrium rates. Theorem 3 shows that XCP equilibrium is constrained max-min fair. It is instructive to compare the XCP equilibrium with the (standard) max-min fair allocation and a class of algorithms proposed in [24].

It is proved in [24] that a (standard) max-min fair rate vector $x^{*}$ is the unique solution of the same hierarchy of problems $\mathbf{P}_{n}$ (8)-(12) defined in Section III, except that the feasible set $X_{0}$ in (7) is replaced with the superset

$$
\bar{X}_{0}:=\left\{x \in \mathbb{R}_{+}^{N} \mid R x \leq c\right\} .
$$

The key feature that results from this much simpler feasible set $\bar{X}_{0}$ is that the bottleneck links under a max-min fair allocation are all fully utilized. Indeed, a rate vector $x^{*} \in \bar{X}_{0}$ is max-min fair if and only if, for every source $i$, there is a link $l \in L(i)$ in its path such that [23]

1) $y_{l}\left(x^{*}\right)=c_{l}$

2) $x_{i}^{*} \geq x_{j}^{*}$ for all $j \in I(l)$.

From Theorem 3, condition 1 is replaced with the fixed point equation $x_{i}^{*}=g_{l}\left(y_{l}\left(x^{*}\right)\right)$ for XCP equilibrium. This more complex condition has several implications.

First, it precludes the much simpler proof techniques used in [24] to show the max-min fair vector is the unique solution of the problems $\mathbf{P}_{n}$.

Second, the algorithm in Section III-D to compute the constrained max-min fair rate vector is more complex than the (centralized) one for the max-min fair vector [24], [23].
Third, and most importantly, the XCP equilibrium can underutilize link capacities and deviate by an arbitrarily large factor from the max-min fair allocation, as we illustrate below.

Max-min fairness is generalized in [24] by restricting the feasible set to a (strict) subset of $\bar{X}_{0}$ in (28). Like XCP, the restriction is specified as additional constraints on source rates $x_{i}$ and link flows $y_{l}$. Like XCP, explicit feedback is required: Each link $l$ feeds back the spare capacity $c_{l}-y_{l}(t)$ to sources that go through this link. Sources adjust their individual rates based on feedback on its path in a way that is distributed, yet avoids overshoot. MaxNet [16] also allows explicit control of link utilization and fairness by separate link and source parameters.

We now illustrate the effect of the additional constraint (7) in $\mathrm{XCP}$ on link utilization and fairness.

As we explained in the proof of Theorem 2, there are three types of links. The first type are bottlenecks for all the flows that go through that link. All links of this type, such as all $l \in L_{1}$ in problem $\mathbf{P}_{1}$, are fully utilized, $y_{l}=c_{l}$. The second type are bottlenecks for none of the flows that go through that link. They are underutilized, $y_{l}<c_{l}$, because the flow rates going through the link are constrained elsewhere. The third type are bottlenecks for some, but not all, of the flows that go through the link. In contrast to the standard max-min fair allocation, these links are also underutilized, $y_{l}<c_{l}$. We can bound the utilization of these partial bottlenecks.

Theorem 6: If $l \in L_{1}(i)$ for some $i$, then

$$
\frac{\alpha}{\gamma \rho_{l}+\alpha} \leq \frac{y_{l}}{c_{l}} \leq 1-\frac{\gamma \sigma_{l}\left(\rho_{l}-\sigma_{l}\right)}{\gamma \rho_{l}+\alpha} .
$$

Proof: Noting that $\rho_{l}<1$ (and that $2\left(\gamma \rho_{l}+\alpha\right)>0$ ), removing the last term from the square root in (23) gives the lower bound

$$
\begin{aligned}
\frac{y_{l}}{c_{l}} & \geq \frac{\alpha+(\gamma+\alpha) \sigma_{l}+\left|\left(\alpha-(\gamma+\alpha) \sigma_{l}\right)\right|}{2\left(\gamma \rho_{l}+\alpha\right)} \\
& \geq \frac{\alpha}{\gamma \rho_{l}+\alpha}
\end{aligned}
$$

where the second inequality is an equality if $\sigma_{l} \leq \alpha /(\gamma+\alpha)$.

To derive the upper bound, first note that $\rho_{l} \geq \sigma_{l}$ from Lemma 2-2. Since $2\left(1-\rho_{l}\right)\left(\gamma \rho_{l}+\alpha\right)>0$ and $\Xi \geq 0$, removing the last term from the square root of (24) yields

$$
\frac{r^{l} N_{l}}{c_{l}} \leq \frac{1-\sigma_{l}}{1-\rho_{l}}-\frac{\gamma \sigma_{l}\left(\rho_{l}-\sigma_{l}\right)}{\left(1-\rho_{l}\right)\left(\gamma \rho_{l}+\alpha\right)} .
$$

Multiplying both sides by $\left(1-\rho_{l}\right)$ and adding $\sigma_{l}$ lead to the upper bound on utilization.

Substituting either $\gamma=0$ or $\rho_{l}=\sigma_{l}$ into either the exact expressions (23) and (24) or the upper and lower bounds (29) and (30) gives full utilization as in the max-min case: $y_{l}=c_{l}$ and $r^{l}=c_{l}\left(1-\sigma_{l}\right) /\left(N_{l}\left(1-\rho_{l}\right)\right)$. This shows that XCP could be made to approach max-min fairness if the bandwidth shuffling were reduced.

On the other hand, link utilization could be arbitrarily low if $\alpha$ and $\gamma$ had been chosen poorly. With the values suggested in [9] however the utilization is at least $80 \%$. Consider a network of two links. Link 1 has $c_{1}=1$ and carries $N_{1}$ flows, while link 2 has $c_{2}=1+\gamma / \alpha$ and carries $N_{2}=N_{1}+1$ flows, consisting of all the traffic on link 1 plus one other flow. As $N_{1} \rightarrow \infty$ we 
get $\rho_{2} \rightarrow 1$. This gives $\sigma_{2}=\alpha /(\gamma+\alpha)$ in the limit. Thus, both terms in the square root of (23) go to zero, whence (29) becomes tight, and $y_{2} / c_{2} \rightarrow 0$ as $\gamma / \alpha \rightarrow \infty$. However, with $\alpha=0.4$ and $\gamma=0.1$ [9], for which positive stability results exist, (23) gives $y_{l} / c_{l}=0.8$.

Similarly, a given flow may obtain an arbitrarily small proportion of its max-min fair bandwidth for any $\alpha>0$ and $\gamma>0$. The ratio of the upper bound on XCP bandwidth (30) to the max-min fair bandwidth, $r^{l, \mathrm{~mm}}=c_{l}\left(1-\sigma_{l}\right) /\left(N_{l}\left(1-\rho_{l}\right)\right)$, is minimized with respect to $\sigma_{l}$ when $\rho_{l}=2 \sigma_{l}-\sigma_{l}^{2}$. Substituting this value into (24) and dividing by $r^{l, \mathrm{~mm}}$ gives

$$
\frac{r^{l}}{r^{l, \mathrm{~mm}}}=\frac{C-\frac{D}{1-\sigma_{l}}+\sqrt{\left[C+\frac{D}{1-\sigma_{l}}\right]^{2}-\frac{E}{1-\sigma_{l}}}}{2\left(\gamma\left(2 \sigma_{l}-\sigma_{l}^{2}\right)+\alpha\right)}
$$

where

$$
\begin{aligned}
& C=\Xi_{l} /\left(1-\sigma_{l}\right)=\gamma \sigma_{l}\left(1-\sigma_{l}\right)+\alpha \\
& D=\gamma \sigma_{l}^{2}\left(2-\sigma_{l}\right) \\
& E=4 \gamma \sigma_{l}^{2} \alpha .
\end{aligned}
$$

Thus

$$
\frac{r^{l}}{r^{l, \mathrm{~mm}}}=\frac{C-\frac{D}{1-\sigma_{l}}+\frac{D}{1-\sigma_{l}} \sqrt{1+\frac{\left(1-\sigma_{l}\right)^{2}}{D^{2}}\left(C^{2}+\frac{2 C D-E}{1-\sigma_{l}}\right)}}{2\left(\gamma\left(2 \sigma_{l}-\sigma_{l}^{2}\right)+\alpha\right)}
$$

Applying the identity $\sqrt{1+x} \leq 1+x / 2$, for $x \geq-1$, gives

$$
\frac{r^{l}}{r^{l, \mathrm{~mm}}} \leq \frac{C+\frac{1-\sigma_{l}}{2 D} C^{2}+\frac{2 C D-E}{2 D}}{2\left(\gamma \sigma_{l}\left(2-\sigma_{l}\right)+\alpha\right)} .
$$

In the limit as $\sigma_{l} \rightarrow 1$, the right-hand side tends to 0 for any $\gamma \neq$ 0 . This demonstrates that, for any nonzero amount of bandwidth shuffling, XCP can be arbitrarily unfair for some topology.

Hence, although the equilibrium of (4) converges to max-min as $\gamma \rightarrow 0$, this convergence is not uniform with respect to topology. In other words, given any topology specified by $(R, c)$, we can choose $\gamma$ sufficiently small so that the resulting allocation is close to max-min fairness. However, for any fixed $\gamma>0$, such as 0.1 used by XCP, there are topologies in which some source rates can be far away from their max-min allocations.

This behavior can be exhibited by a simple two-link network: One link has capacity 1 and carries $n^{2}$ flows, while the other carries $n^{2}-1$ of those same flows and has capacity $(n-1) / n$. This network has $\sigma_{2}=(n-1) / n$ and $\rho_{2}=\left(n^{2}-1\right) / n^{2}=$ $2 \sigma_{2}-\sigma_{2}^{2}$. Hence, $\sigma_{2} \rightarrow 1$ as $n \rightarrow \infty$ and $r^{2} / r^{2, \mathrm{~mm}} \rightarrow 0$.

These asymptotic results will be illustrated and confirmed by simulation in the following section.

\section{Simulation Results}

This section presents simulation results using the implementation available from [9] for NS-2 [25]. These results verify the accuracy of our algorithm in Section III-D and confirm our qualitative discussion in Section IV on the utilization and fairness properties of XCP.

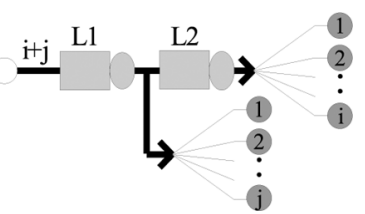

Fig. 1. Topology for Scenarios 1 and 2.

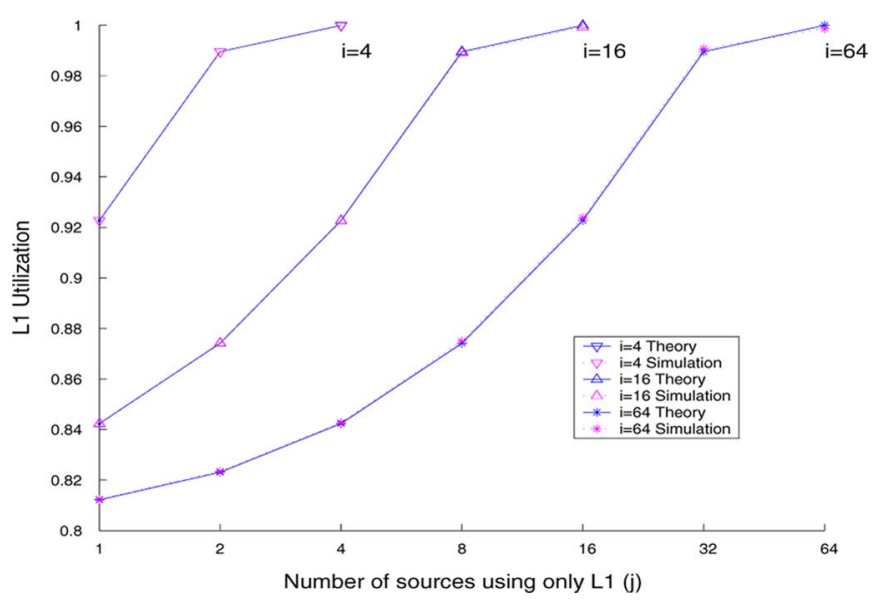

Fig. 2. Scenario 1: Utilization.

All sources always have packets to send. Links are randomly assigned delays between 5 and $500 \mathrm{~ms}$, uniformly on a log scale. Results for different realisations were indistinguishable. The XCP default parameters $\alpha=0.4, \beta=0.226$ and $\gamma=0.1$ are used. Although the analysis neglects the "residual" terms, the simulations include them. However, as remarked in Section II-B, they have minimal impact on equilibrium properties, yielding a good match between theory and simulation. Although XCP has been found to be unstable in specific circumstances [26], all of the simulations here exhibited stable dynamics.

The topology used for Scenarios 1 and 2 is shown in Fig. 1 and consists of two links, with $i+j$ sources traversing link L1 and $j$ sources traversing L2.

Using protocols that have nonempty queues in eqiulibrium, flows in each direction are coupled through changes in the RTT and a process known as ACK compression. Since XCP maintains empty queues at eqiulibrium, it is sufficient to consider unidirectional flows, which we do here for clarity.

Scenario 1 investigates the utilization of L1 as the number of sources traversing L1 and L2 is changed. In the experiment $i \geq j$, with $c_{1}=155 \mathrm{Mbps}$ and $c_{2}=100 \mathrm{Mbps}$. The utilization of L1 for a range of $i$ and $j$ is shown in Fig. 2. A max-min fair allocation would result in a full utilization of L1 for all $i$ and $j$ combinations. However, as the number of sources bottlenecked at L2 increases, XCP's utilization of L1 decreases.

Since XCP's "residual" terms depend on feedback from upstream nodes, the equilibrium rates depend on the order in which links are traversed. If the direction of flow in this network were reversed, then the utilization would be $0 \%-4 \%$ higher than for the case considered and than the theoretical predictions.

Scenario 2 demonstrates that XCP can be arbitrarily unfair for some topology. Let $c_{1}=155 \mathrm{Mbps}, c_{2}=c_{1}(n-1) / n, i=$ 


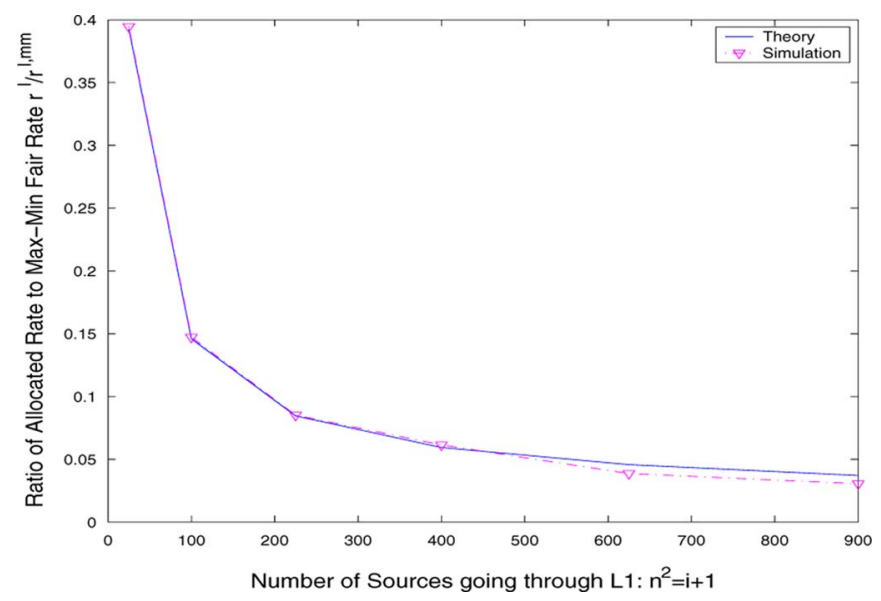

Fig. 3. Scenario 2: Unfairness, $r^{2} / r^{2, \mathrm{~mm}} \rightarrow 0$ as $n \rightarrow \infty$.

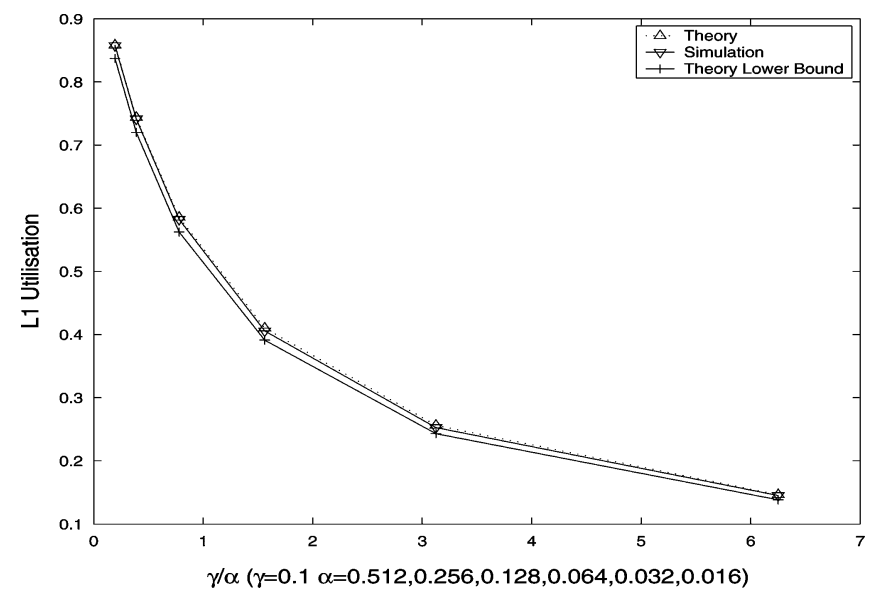

Fig. 4. Scenario 3: Utilization of L1 as function of $\gamma / \alpha$.

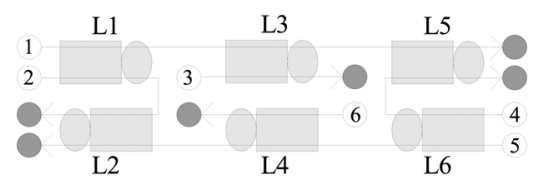

Fig. 5. Scenario 3 topology.

$n^{2}-1$ and $j=1$. The ratio of the rate of the source traversing only L1 to the max-min fair rate is plotted in Fig. 3. Indeed the unfairness increases with the number of sources in the network, confirming the theory.

Scenario 3 studies XCP with nonstandard parameters. It verifies that $y_{2} / c_{2} \rightarrow 0$ as $\gamma / \alpha \rightarrow \infty$. We set $c_{2}=200 \mathrm{Mbps}$, $c_{1}=c_{2}(1+\gamma / \alpha), i=256$ and $j=1$. The parameter $\alpha$ is varied from 0.512 to 0.016 and the utilisation of $\mathrm{L} 1$ as a function of $\gamma / \alpha$, as well as the lower bound from (29), are plotted in Fig. 4.

Scenario 4 tests the rate allocation algorithm for a more complicated topology as shown in 5. The link capacities in Mbps are $c_{1}=10, c_{3}=8, c_{4}=8, c_{5}=7, c_{6}=6$ and $c_{2}$ is varied in this experiment. Delay is set to $d_{l}=10 \mathrm{~ms}$. The source rates are plotted in Fig. 6. There is a good agreement between the predicted and measured rates even though the lower bandwidth delay product makes the fluid flow approximation more questionable.

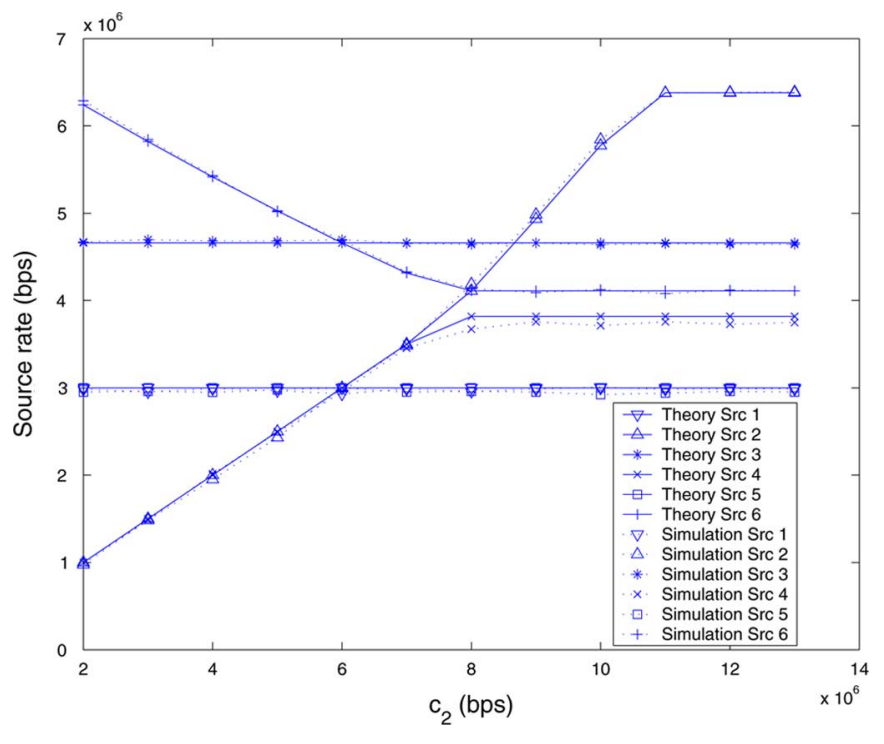

Fig. 6. Scenario 4: Throughputs.

\section{CONCLUSION}

We have presented a dynamic model of XCP and used it to completely characterize its equilibrium properties. We have shown that XCP clears the queues in equilibrium and has unique equilibrium rates that solve a constrained max-min fairness problem. The additional constraint under XCP can lead to unfairness for some network topologies. XCP gives a utilization of at least $80 \%$, but a poor choice of $\alpha$ or $\gamma$ could lead to arbitrarily low utilization. We have provided an algorithm to compute the equilibrium for general networks, and have presented simulation results to illustrate these findings.

An important question that we have not pursued is the dynamic properties of XCP, such as its stability. Even though the "residual" terms in the XCP code do not seem to affect equilibrium properties drastically, they may be important in determining its dynamic properties and, hence, should be taken into account in such an analysis. It is important to understand the stability of individual source rates $x_{i}(t)$, in addition to the aggregate rate $y_{l}(t)$, as studied in [9], in general networks in the presence of nonuniform delay. Since equilibrium queues are zero, the usual practice of linearizing around the equilibrium needs caution at the tightest bottlenecks that have zero queue yet full utilization.

\section{APPENDIX A VALID EQUILIBRIUM RATE}

Proof (Lemma 2):

1) By Lemma $1, F_{l i}>0$ and $F_{l j}=0$. This implies

$$
\frac{h_{l}+\phi_{l}^{+}}{N_{l} x_{i}}>\frac{h_{l}+\phi_{l}^{-}}{y_{l}}=\frac{h_{l}+\phi_{l}^{+}}{N_{l} x_{j}} .
$$

2) If this were not the case, then the average rate of flows in $I_{0}(l)$ (and hence the rate of at least one such flow) would be greater than $c_{l} / N_{l}$. Part 1 would then require the total rate to exceed the capacity. 
3) From part 1

$$
\begin{aligned}
N_{l} r^{l} & =N_{l 1} r^{l}+N_{l 0} r^{l} \\
& \geq N_{l 1} r^{l}+y_{l 0}=y_{l}
\end{aligned}
$$

with equality if and only if $y_{l 0}=0$.

4) Otherwise, $h_{l}=0$ and $\phi_{l}=0$ since $I_{1}(l) \neq \emptyset$ (by

Lemma 1, part 4). Then, $y_{l}=c_{l}$ and $b_{l}=0$, whence $h_{l}=\max \left(\gamma d_{l} c_{l}-0,0\right)>0$.

5) $\sigma_{l}=y_{l 0} / c_{l} \leq y_{l} / c_{l}$ with equality if and only if $y_{l 0}=y_{l}$.

Lemma 8: The smaller solution to (26) does not give a valid equilibrium rate for link $l$.

Proof: For a rate, $y_{l}$, to be valid requires $y_{l} / c_{l}>\sigma_{l}$, by Lemma 2(5).

Let $\theta=(1+\gamma / \alpha) \sigma_{l}$ and $\psi=\left(1+\rho_{l} \gamma / \alpha\right) \sigma_{l} \leq \theta$. Then, dividing the numerator and denominator of (26) by $\alpha$ gives

$$
\frac{y_{l}}{c_{l}}=\frac{1+\theta \pm \sqrt{(1+\theta)^{2}-4 \psi}}{2 \psi / \sigma_{l}} .
$$

If the lower root were valid, then

$$
1+\theta-2 \psi>\sqrt{(1+\theta)^{2}-4 \psi}
$$

whence

$$
(1+\theta)^{2}-4(1+\theta) \psi+4 \psi^{2}>(1+\theta)^{2}-4 \psi .
$$

However, $0 \leq \psi \leq \theta$, which yields a contradiction. Thus, the lower root is not valid.

\section{APPENDIX B}

\section{PROPERTIES OF OPTIMIZATION PROBLEMS}

Proof (Lemma 4): Note that $g_{l}(x)=g_{l}\left(y_{l}\right)$ depends on $x$ only through $y_{l}$. Now, $x \in X_{n}$ are all of the form

$$
x_{i}= \begin{cases}r_{m}, & i \in I_{m}, m \leq n \\ r_{n}+\epsilon_{i}, & i \notin \bar{I}_{n}\end{cases}
$$

where $\epsilon_{i}>0$. Write $x \in X_{n}$ as $x\left(\epsilon_{i}, i \notin \bar{I}_{n}\right)$. Hence, we can write $y_{l}$ also as a function of $\left(\epsilon_{i}, i \notin \bar{I}_{n}\right)$

$$
\begin{aligned}
& y_{l}\left(\epsilon_{i}, i \notin \bar{I}_{n}\right) \\
& =\sum_{m=1}^{n} r_{m} \sum_{i \in I_{m}} R_{l i}+\sum_{i \notin \bar{I}_{n}} R_{l i}\left(r_{n}+\epsilon_{i}\right) \\
& =\sum_{m=1}^{n-1} r_{m} \sum_{i \in I_{m}} R_{l i}+r_{n} \sum_{i \notin \bar{I}_{n-1}} R_{l i}+\sum_{i \notin \bar{I}_{n}} R_{l i} \epsilon_{i} .
\end{aligned}
$$

Given any $\left(\epsilon_{i}, i \notin \bar{I}_{n}\right)$, define the average $\epsilon$ by

$$
\epsilon \cdot \sum_{i \notin \bar{I}_{n-1}} R_{l i}=\sum_{i \notin \bar{I}_{n}} R_{l i} \epsilon_{i}
$$

and consider the vector $\left(\epsilon_{i}=\epsilon, i \notin \bar{I}_{n}\right)$ with equal components. From (33), this vector produces the same link flow $y_{l}$. Moreover, $x(\epsilon):=x\left(\epsilon_{i}=\epsilon, i \notin \bar{I}_{n}\right)$ defined by this vector also satisfies $x_{i}(\epsilon) \leq g_{l}\left(y_{l}\right)$ for all $i \in I(l)$, and hence is in $X_{n}$. This is because for $i \in I_{m}, m \leq n, x_{i}(\epsilon)=r_{m} \leq g_{l}\left(y_{l}\right)$ since the original $x\left(\left(\epsilon_{i}, i \notin \bar{I}_{n}\right)\right)$ is in $X_{n}$. For $i \notin \bar{I}_{n}$

$$
x_{i}(\epsilon)=r_{n}+\epsilon \leq r_{n}+\max _{i} \epsilon_{i} \leq g_{l}\left(y_{l}\right)
$$

where the first inequality follows because $\epsilon$ is the average of $\epsilon_{i}$, and the last inequality follows because the original $x\left(\epsilon_{i}, i \notin\right.$ $\left.\left.\bar{I}_{n}\right)\right)$ is in $X_{n}$. Hence, if $\left(\epsilon_{i}^{*}, i \notin \bar{I}_{n}\right)$ achieves the maximum in (9), the vector $\left(\epsilon_{i}^{*}=\epsilon^{*}, i \notin \bar{I}_{n}\right)$ with a common value $\epsilon^{*}$ also achieves the maximum.

Proof (Lemma 5): Define

$$
f(\epsilon):=\hat{g}_{l}(\epsilon)-(s+\eta \epsilon)
$$

We will show that there exists $\epsilon_{l} \geq 0$, with equality if and only if $s=\hat{g}_{l}(0)$, such that $f\left(\epsilon_{l}\right)=0$ under the conditions given in the lemma. Consider the two cases separately.

Case 1: $s \leq \hat{g}_{l}(0)$. If $s=\hat{g}_{l}(0)$ then $f(0)=0$ and $\epsilon_{l}=0$. Otherwise, $f(0)>0$ and it remains to show that there exists a suitable $\epsilon_{l}>0$. Consider

$$
\begin{aligned}
\lim _{\epsilon \rightarrow \infty} f(\epsilon)= & \lim _{\epsilon \rightarrow \infty} \hat{g}_{l}(\epsilon)-(s+\eta \epsilon) \\
= & \lim _{\epsilon \rightarrow \infty} \frac{\gamma(z+\eta \epsilon)^{2}}{N_{l}\left((\gamma+\alpha)(z+\eta \epsilon)-\alpha c_{l}\right)}-(s+\eta \epsilon) \\
= & \lim _{\epsilon \rightarrow \infty} \epsilon \\
& \cdot\left(\lim _{\epsilon \rightarrow \infty} \frac{\gamma(z / \epsilon+\eta)^{2}}{N_{l}\left((\gamma+\alpha)(z / \epsilon+\eta)-\alpha c_{l} / \epsilon\right)}\right. \\
& -(s / \epsilon+\eta)) \\
= & \lim _{\epsilon \rightarrow \infty} \epsilon \cdot \eta\left(\frac{1}{N_{l}} \frac{\gamma}{\gamma+\alpha}-1\right) \\
= & -\infty
\end{aligned}
$$

since $N_{l} \geq 1$. This implies that there exists $\epsilon_{1}$ sufficiently large such that $f\left(\epsilon_{1}\right)<0$. Since $f$ is continuous on $\left[0, \epsilon_{1}\right]$, there exists an $\epsilon_{l} \in\left(0, \epsilon_{1}\right)$ such that $f\left(\epsilon_{l}\right)=0$. This is illustrated in Fig. 7.

Case 2: $\hat{g}_{l}(0)<0$. Then

$$
z<\frac{\alpha c_{l}}{\gamma+\alpha}
$$

and hence

$$
\bar{\epsilon}:=\frac{1}{\eta}\left(\frac{\alpha c_{l}}{\gamma+\alpha}-z\right)>0 .
$$

Moreover, as $\epsilon \rightarrow \bar{\epsilon}$ from above, $\hat{g}_{l}(\epsilon) \rightarrow+\infty$. Since $\hat{g}_{l}(\epsilon)$ is continuous for $\epsilon>\bar{\epsilon}$, there exists $\epsilon_{0}>$ $\bar{\epsilon}$ such that $s+\eta \epsilon_{0}<\hat{g}_{l}\left(\epsilon_{0}\right)$, i.e., $f\left(\epsilon_{0}\right)>0$. The same argument as in Case 1 shows that there exists an $\epsilon_{1}$ with $f\left(\epsilon_{1}\right)<0$. Since $\hat{g}_{l}$ is continuous on $\left[\epsilon_{0}, \epsilon_{1}\right]$, there exists $\epsilon_{l} \in\left(\epsilon_{0}, \epsilon_{1}\right)$ where $f\left(\epsilon_{l}\right)=0$.

We now prove that $\epsilon_{l}$ is unique. The argument also shows that over $\left\{\epsilon \mid \hat{g}_{l}(\epsilon)>0\right\}, s+\eta \epsilon>\hat{g}_{l}(\epsilon)$ if and only if $\epsilon>\epsilon_{l}$. First note that $\bar{\epsilon}$ may be negative. They key observation, illustrated in Fig. 7, is that

1) for $\epsilon<\bar{\epsilon}, \hat{g}_{l}(\epsilon)$ is negative, concave, and approaches $-\infty$ as $\epsilon \rightarrow \bar{\epsilon}$ from below; 


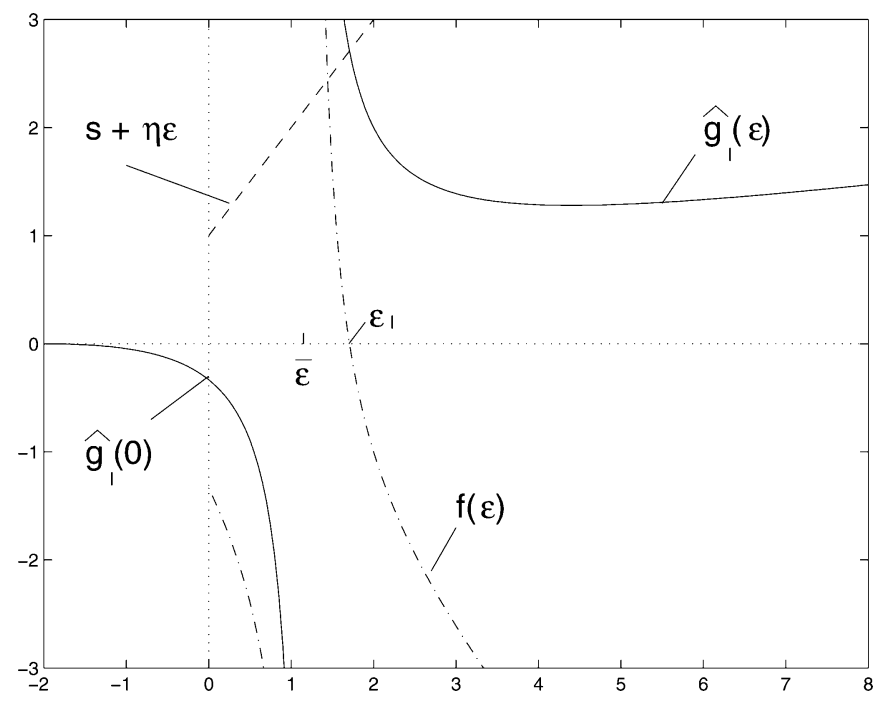

Fig. 7. $\hat{g}_{l}(\epsilon)$ in (18) and $f(\epsilon)=\hat{g}_{l}(\epsilon)-(s+\eta \epsilon)$.

2) for $\epsilon>\bar{\epsilon}, \hat{g}_{l}(\epsilon)$ is positive, convex, attains its minimum at

$$
\epsilon=\frac{1}{\eta}\left(\frac{2 \alpha c_{l}}{\gamma+\alpha}-z\right)
$$

and approaches $+\infty$ as $\epsilon \rightarrow \bar{\epsilon}$ from above.

A moment of thought then convinces one that it suffices to show that $\hat{g}_{l}(\epsilon)$ grows less rapidly than $s+\eta \epsilon$, i.e.,

$$
\frac{d \hat{g}_{l}}{d \epsilon}<\eta
$$

Letting $y_{l}=z+\eta \epsilon$, we have

$$
\frac{d \hat{g}_{l}}{d \epsilon}=\frac{\eta}{N_{l}} \cdot \frac{\gamma y_{l}\left((\gamma+\alpha) y_{l}-2 \alpha c_{l}\right)}{\left((\gamma+\alpha) y_{l}-\alpha c_{l}\right)^{2}} .
$$

Since $N_{l} \geq 1$, if (34) is violated, the above implies that

$$
\frac{\gamma y_{l}\left((\gamma+\alpha) y_{l}-2 \alpha c_{l}\right)}{\left((\gamma+\alpha) y_{l}-\alpha c_{l}\right)^{2}} \geq 1
$$

which implies

$$
\alpha^{2}\left(y_{l}+c_{l}\right)^{2}+\alpha \gamma y_{l}^{2} \leq 0
$$

which is a contradiction since $c_{l}, \alpha>0$ and $\gamma, y_{l} \geq 0$. Hence, (34) must hold and $s+\eta \epsilon>g_{l}(\epsilon)$ if and only if $\epsilon>\epsilon_{l}$ whenever $g_{l}(\epsilon)>0$.

Proof (Lemma 6): The first assertion follows directly from the definitions of $L_{n}, I_{n}$ and $\hat{X}_{n}$ in (10), (11) and (15), respectively. We will prove the second assertion by induction on $n$.

Base case $n=1$ : Fix any $x \in \hat{X}_{1}$.

Consider an $l \notin L_{1}=\bar{L}_{1}$. By (13), $c_{l} / N_{l}>r_{1}$. Now

$$
x_{i}(\epsilon ; 1)= \begin{cases}r_{1} & i \in I_{1} \\ r_{1}+\epsilon & i \notin I_{1}\end{cases}
$$

Hence

$$
g_{l}(x(0 ; 1))=\frac{\gamma\left(N_{l} r_{1}\right)^{2}}{N_{l}\left((\gamma+\alpha) N_{l} r_{1}-\alpha c_{l}\right)} .
$$

If

$$
N_{l} r_{1}<\frac{\alpha}{\gamma+\alpha} c_{l}
$$

then $g_{l}(x(0 ; 1))<0$. Otherwise, $g_{l}(x(0 ; 1))>0$. We claim $x_{i}(0,1)=r_{1}<g_{l}(x(0 ; 1))$ for all $i \in I(l)$. If not, then

$$
r_{1} \geq \frac{\gamma\left(N_{l} r_{1}\right)^{2}}{N_{l}\left((\gamma+\alpha) N_{l} r_{1}-\alpha c_{l}\right)}
$$

yielding $N_{l} r_{1} \geq c_{l}$, a contradiction. Hence, if $l \notin \bar{L}_{1}$, then either $g_{l}(x(0 ; 1))<0$ or $x_{i}(0 ; 1)<g_{l}(x(0 ; 1))$ for all $i \in I(l)$.

Induction hypothesis: Suppose the second assertion holds for $n$. We will prove it for $n+1$.

Induction: Fix an $l \notin \bar{L}_{n+1}$. First note that $l \notin \bar{L}_{n}$. Consider all links $\hat{l} \notin \bar{L}_{n}$. By the induction hypothesis, either $g_{\hat{l}}(x(0 ; n))<0$ or $x_{i}(0 ; n)<g_{\hat{l}}(x(0 ; n))$ for all $i \in I(\hat{l})$. For an $i \notin \bar{I}_{n}$, we have from (10), for $\epsilon \geq 0$

$$
\begin{aligned}
& x_{i}(\epsilon ; n)=r_{n}+\epsilon \\
& y_{\hat{l}}(\epsilon ; n)=z_{\hat{l}}+\eta_{\hat{l}} \epsilon_{\hat{l}}
\end{aligned}
$$

where, from (17)

$$
\begin{aligned}
z_{\hat{l}} & :=\sum_{m=1}^{n} r_{m} \sum_{i \in I_{m}} R_{\hat{l} i}+r_{n} \sum_{i \notin \bar{I}_{n}} R_{\hat{l} i} \\
\eta_{\hat{l}} & :=\sum_{i \notin \bar{I}_{n}} R_{\hat{l} i} .
\end{aligned}
$$

From (18), $\hat{g}_{\hat{l}}(0)=g_{\hat{l}}\left(z_{\hat{l}}\right)=g_{\hat{\imath}}(x(0 ; n))<0$, and hence the induction hypothesis implies that either $\hat{g}_{\hat{l}}(0)<0$ or $r_{n}<$ $\hat{g}_{\hat{l}}(0)$. Lemma 5 then implies that, for each $\hat{l} \notin \bar{L}_{n}$, there exists a unique $\epsilon_{\hat{l}}>0$ such that $r_{n}+\epsilon_{\hat{l}}=\hat{g}_{\hat{l}}\left(\epsilon_{\hat{l}}\right)=g_{\hat{l}}\left(z_{\hat{l}}+\eta_{\hat{l}} \epsilon_{\hat{l}}\right)$. The minimum $g_{\hat{l}}\left(z_{\hat{l}}+\eta_{\hat{l}} \hat{\epsilon}_{\hat{l}}\right)$ over $\hat{l} \notin \bar{L}_{n}$ is $r_{n+1}$, and these minimizing $\hat{l}$ constitute $L_{n+1}$. All the sources $i \notin \bar{I}_{n}$ that go through a link in $L_{n+1}$ are assigned the common rate $r_{n+1}$, and they are collected into $X_{n+1}$.

Since $l \notin \bar{L}_{n}$ and $l \notin L_{n+1}$, the corresponding $\epsilon_{l}$ satisfies

$$
\begin{aligned}
& r_{n}+\epsilon_{l}=g_{l}\left(z_{l}+\eta_{l} \epsilon_{l}\right) \\
& r_{n}+\epsilon_{l}>r_{n+1}=r_{n}+\min _{\hat{l} \notin \bar{L}_{n}} \epsilon_{\hat{l}} .
\end{aligned}
$$

However, $x_{i}(\epsilon ; n+1)=r_{n+1}+\epsilon$ for all $i \notin \bar{I}_{n+1}$. Hence (35) and Lemma 5-2 (with $z=y_{l}(0 ; n), s=r_{n}, \eta=\sum_{i \notin \bar{I}_{n}} R_{l i}$ ) imply that either $g_{l}(x(0 ; n+1)) \leq 0$ or $x_{i}(0 ; n+1)=r_{n}+$ $\min _{\hat{l} \in \bar{L}_{n}} \epsilon_{\hat{l}}<\hat{g}_{l}\left(\min _{\hat{l} \in \bar{L}_{n}} \epsilon_{\hat{l}}\right)=g_{l}(x(0 ; n+1))$. The proof is completed by noting that $g_{l}(x(0 ; n+1)) \neq 0$, since $y_{l} \geq r_{1}>$ 0 .

\section{APPENDIX C \\ CORRECTNESS OF RATE ALGORITHM}

To show the correctness of the algorithm in Section III-D to find XCP's equilibrium rates, it suffices to show that the results satisfy the two conditions stated in Section III-B.

Each source $i$ is assigned a rate in the same step as a particular link $l$. If $r^{l}$ is chosen according to (24) with the true equilibrium 
values of $\rho_{l}$ and $\sigma_{l}$, then $F_{l i}=0$, since that is the condition from which (24) was derived. Similarly, (24) implies $y_{l} \leq c_{l}$, as established in the proof of Theorem 6.

The correctness of the algorithm can thus be established by showing that the true equilibrium values of $\sigma_{l}$ and $\rho_{l}$ are used when the final value $r^{l}$ is calculated in step 2.4.1. For each link $l$, the values of $\sigma_{l}$ and $\rho_{l}$ depend only on network parameters and flows $i$ with rates $x_{i}<r^{l}$, by Lemma 2-1. That is, if the rates selected by the algorithm are such that the rate $r^{l}$ of each link $l$ is greater than the rates of the flows flowing through $l$ but bottlenecked elsewhere, then the rates must form an equilibrium of XCP. The theorem then results from the following lemma.

Lemma 9: For each $l$ and $n$ for which $r^{l}(n+1)$ is defined, $r^{l}(n+1) \geq r_{n}$. Moreover, if $\gamma<\alpha$, then for each $l$ and $n$ for which $r^{l}(n+1)$ is defined, $r^{l}(n+1) \geq r^{l}(n)$.

Proof: Consider an arbitrary link, $l$, and iteration, $n$. If $l$ does not carry any flows in $\Delta(n)$, then $r^{l}(n+1)=r^{l}(n)>r_{n}$ as required. Consider now the case that $l$ does carry a flow in $\Delta(n)$.

Let $x=r_{n} N_{l} / c_{l}$ be the rate of flows allocated in iteration $n$, normalized to link $l$. Let $\rho(u)=\rho_{l}(n)+u$ and $\sigma(u)=\sigma_{l}(n)+$ $u x$. Then $\rho(0)$ and $\sigma(0)$ are the fraction of allocated flows and allocated capacity on link $l$ before step 2.4 .1 of iteration $n$, while $\rho(\delta)$ and $\sigma(\delta)$ are the values after the update, where $\delta=\rho_{l}(n+$ 1) $-\rho_{l}(n)>0$.

Let $z(u)$ be the value of $r^{l} N_{l} / c_{l}$ calculated from (27) using $\rho(u)$ and $\sigma(u)$. Note that $z \geq 1$ by Lemma 2-3, since $c_{l} \geq y_{l}$. Moreover, $x<z(0)$, since if $z(0) \leq x$ then link $l$ would have been an element of $M(n)$ and $r^{l}(n+1)$ would not be defined. To prove the lemma, it is sufficient to show that $z(\delta) \geq x$ and that if $\gamma>\alpha$ then $d z / d u \geq 0$.

Below, the argument $(u)$ will be dropped when no ambiguity can arise. Differentiating (27) with respect to $u$, and noting that $z(u)$ is the larger of the two solutions of (27), gives

$$
\begin{aligned}
0 & =\frac{d A_{l}}{d u} z^{2}+2 z A_{l} \frac{d z}{d u}+\frac{d B_{l}}{d u} z+B_{l} \frac{d z}{d u}+\frac{d C_{l}}{d u} \\
& =\sqrt{B_{l}^{2}-4 A_{l} C_{l}} \frac{d z}{d u}+\frac{d A_{l}}{d u} z^{2}+\frac{d B_{l}}{d u} z+\frac{d C_{l}}{d u} .
\end{aligned}
$$

Now

$$
\begin{aligned}
\frac{d A_{l}}{d u} z^{2}+\frac{d B_{l}}{d u} z+\frac{d C_{l}}{d u} \\
=[\gamma(1-\rho)-(\gamma \rho+\alpha)] z^{2} \\
\quad+[2 \gamma \sigma-\gamma x(1-\rho)+x(\gamma \rho+\alpha)] z-2 \gamma \sigma x \\
=(x-z)[z(\alpha-\gamma)+2 \gamma(z \rho-\sigma)]
\end{aligned}
$$

giving

$$
\frac{d z}{d u}=(z-x) \frac{[z(\alpha-\gamma)+2 \gamma(z \rho-\sigma)]}{\sqrt{B_{l}^{2}-4 A_{l} C_{l}}} .
$$

If $\gamma<\alpha$, then $d z / d u>0$, and $z$ remains greater than $x$. This is because $2 \gamma(z \rho-\sigma) \geq 0$, since $z \geq 1$ and $0 \leq \sigma \leq \rho$. This establishes the second part of the lemma.

If $\gamma \geq \alpha$, then the right-hand side need not be positive. However, the second factor is bounded, and so $z$ approaches $x$ expo- nentially as $u$ increases, and so can never drop below $x$. In particular, $z(\delta)>x$, which establishes the first part of the lemma.

\section{ACKNOWLEDGMENT}

The authors would like to thank D. Katabi of Massachusetts Institute of Technology (MIT), Cambridge, for helpful discussions.

\section{REFERENCES}

[1] V. Jacobson, "Congestion avoidance and control," in Proc. ACM SIGCOMM, Aug. 1988, pp. 314-329.

[2] C. Hollot, V. Misra, D. Towsley, and W. Gong, "Analysis and design of controllers for AQM routers supporting TCP flows," IEEE Trans. Autom. Control, vol. 47, no. 6, pp. 945-959, Jun. 2002.

[3] S. H. Low, F. Paganini, J. Wang, and J. C. Doyle, "Linear stability of TCP/RED and a scalable control," Comput. Netw. J., vol. 43, no. 5, pp. 633-647, 2003.

[4] C. Casetti, M. Gerla, S. Mascolo, M. Sansadidi, and R. Wang, "TCP Westwood: End-to-end congestion control for wired/wireless networks," Wireless Netw. J., vol. 8, pp. 467-479, 2002.

[5] S. Floyd, "High-speed TCP for large congestion windows," IETF, RFC 3649, Dec. 2003.

[6] D. X. Wei, C. Jin, and S. H. Low, "FAST TCP: Motivation, architecture, algorithms, performance," IEEE/ACM Trans. Netw., vol. 14, no. 6, pp. $1246-1259$, Dec. 2006

[7] I. Rhee and L. Xu, "CUBIC: A new TCP-friendly high-speed TCP variant," presented at the PFLDnet, 2005.

[8] D. Leith and R. Shorten, "H-TCP: TCP for high-speed and long-distance networks," presented at the PFLDnet, Argonne, IL, 2004, in Proc.

[9] D. Katabi, M. Handley, and C. Rohrs, "Congestion control for highbandwidth delay product networks," in Proc. ACM SIGCOMM, 2002.

[10] A. Falk, D. Katabi, and Y. Pryadkin, "Specification for the Explicit Control Protocol (XCP)," draft-falk-xcp-03.txt, 2007.

[11] Y. Zhang and M. Ahmed, "A control theoretic analysis of XCP," in Proc. IEEE GLOBECOM, Mar. 2005, pp. 2831-2835.

[12] F. Abrantes and M. Ricardo, "XCP for shared access multirate media," Comput. Commun. Rev., vol. 36, pp. 29-38, Jul. 2006.

[13] Y. Sakumoto, H. Ohsaki, and M. Imase, "On XCP stability in a heterogeneous network," in Proc. Int. Symp. Comput. Commun., 2007, pp. 531-537.

[14] C. Wilson, C. Coakley, and B. Y. Zhao, "Fairness attacks in the explicit control protocol," in Proc. IEEE IWQoS, 2007, pp. 21-28.

[15] Y. Zhang and T. Henderson, "An implementation and experimental study of the explicit control protocol (XCP)," in Proc. IEEE INFOCOM, Miami, FL, 2005, pp. 1037-1048.

[16] B. Wydrowski and M. Zukerman, "MaxNet: A congestion control architecture for max-min fairness," IEEE Commun. Lett., vol. 6, no. 11, pp. 512-514, Nov. 2002.

[17] B. Wydrowski, L. L. H. Andrew, and M. Zukerman, "MaxNet: A congestion control architecture for scalable networks," IEEE Commun. Lett., vol. 7, no. 10, pp. 511-513, Oct. 2003.

[18] H. Balakrishnan, N. Dukkipati, N. McKeown, and C. J. Tomlin, "Stability analysis of explicit congestion control protocols," IEEE Commun. Lett., vol. 11, no. 10, pp. 823-825, Oct. 2007.

[19] S. Low, L. Andrew, and B. Wydrowski, "Understanding XCP: Equilibrium and fairness," in Proc. IEEE INFOCOM, 2005, pp. 1025-1036.

[20] P. Wang and D. L. Mills, "Further analysis of XCP equilibrium performance," in Proc. IEEE Globecom, 2006, pp. 1-5.

[21] J. M. Jaffe, "Bottleneck flow control," IEEE Trans. Commun., vol COM-29, no. 7, pp. 954-962, Jul. 1981.

[22] S. Athuraliya, V. H. Li, S. H. Low, and Q. Yin, "REM: Active queue management," IEEE Network, vol. 15, pp. 48-53, May/June 2001.

[23] D. Bertsekas and R. Gallager, Data Networks, 2nd ed. Englewood Cliffs, NJ: Prentice-Hall, 1992

[24] E. M. Gafni and D. P. Bertsekas, "Dynamic control of session input rates in communication networks," IEEE Trans. Autom. Control, vol. AC-29, no. 1, pp. 1009-1016, Jan. 1984

[25] NS Network Simulator, [Online]. Available: http://www.isi.edu/ nsnam/ns/

[26] L. L. H. Andrew, B. P. Wydrowski, and S. H. Low, "An example of instability in XCP," [Online]. Available: http://netlab.caltech.edu/lachlan/abstract/xcpInstability.pdf, unpublished 


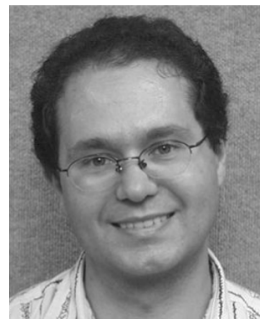

Lachlan L. H. Andrew (M'97-SM'05) received the B.Sc. degree in computer science, the B.E. degree in electrical engineering, and the Ph.D. degree in engineering from the University of Melbourne, Australia, in 1992, 1993, and 1997, respectively.

Since 2008, he has been an Associate Professor at Swinburne University of Technology, Hawthorn, Australia. From 2005 to 2008, he was a Senior Research Engineer in the Department of Computer Science at California Institute of Technology, Pasadena. Prior to that, he was a Senior Research Fellow at the University of Melbourne and a lecturer at RMIT University, Melbourne, Australia. His research interests include performance analysis of congestion control, resource allocation algorithms and energy-efficient networking.

Dr. Andrew is a Member of the IET and the Association for Computing Machinery. He was co-recipient of the Best Paper Award at IEEE MASS'07.

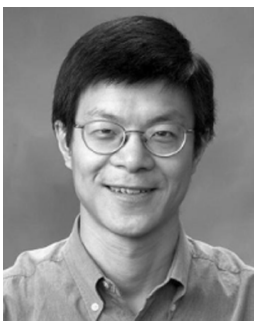

Steven H. Low (M'92-SM'99-F'08) received the B.S. degree from Cornell University, Ithaca, NY, and the Ph.D. degree from the University of California at Berkeley.

$\mathrm{He}$ is a Professor of the Computer Science and Electrical Engineering Departments at California Institute of Technology, Pasadena, and an Adjunct Professor of the Swinburne University of Technology, Hawthorn, Australia.

Dr. Low was a co-recipient of the IEEE William R. Bennett Prize Paper Award in 1997 and the 1996 R\&D 100 Award. He was on the Editorial Board of the IEEE/ACM TRANSACTIONS ON NETWORKING from 1997 to 2006 and Computer Networks Journal from 2003 to 2005. He is on the Editorial Boards of Computing Surveys and Foundations and Trends in Networking. He is a Senior Editor of the IEEE JOURNAL ON SELECTED AREAS IN COMMUNICATIONS and a Co-Editor of the Springer book series on Optimization and Control of Communication Systems: Theory and Applications. He was a Member of the Networking and Information Technology Technical Advisory Group for the U.S. Presidents Council of Advisors on Science and Technology (PCAST).

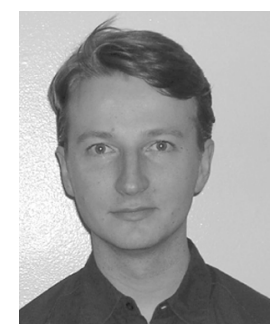

Bartek P. Wydrowski (M'03) received the B.E. degree in electrical engineering and the Ph.D. degree in engineering from the University of Melbourne, Australia, in 1998 and 2003, respectively.

Since 2007, he has been a Software Engineer at Google, New York, NY, prior to which he worked at FastSoft Inc., Pasadena, CA, and as a Research Engineer at California Institute of Technology, Pasadena, between 2004 and 2005 .

Dr. Wydrowski was awarded a 2003 Victoria Fellowship. 